\title{
INFLUENCE DE LA MISE A L'HERBE DES VACHES LAITIÈRES SUR LES INDICES DE LA MATIÈRE GRASSE DU BEURRE ET SUR LES TENEURS EN DIFFÉRENTS ACIDES GRAS POLY-INSATURÉ́,
}

\author{
Simone KUZDZAL-SAVOIE et W. KUZDZAL \\ Station centrale de Recherches laitières \\ et de Technologie des produits animaux, \\ Centre national de Recherches zootechniques, Joty-en-Josas.
}

\section{SOMMAIRE}

Au cours de trois printemps successifs (1957, 1958 et 1959) et pendant une période d'une quinzaine de jours encadrant la mise à l'herbe des vaches laitières, on a déterminé les indices d'iode et de saponification, l'acidité volatile et la composition des acides poly-insaturés de la matière grasse du beurre.

Le beurre était fabriqué chaque jour, à partir du lait produit matin et soir par différents groupes de vaches laitières appartenant au Centre National de Recherches Zootechniques de Jouy-en-Josas.

Le passage du régime de stabulation au régime de pâturage entraîne les modifications suivantes: augmentation de l'indice d'iode, diminution de l'indice de saponification et de l'acidité volatile, augmentation des teneurs en acides diènes conjugués et triènes non conjugués (linolénique). La teneur en acide linoléique s'élève passagèrement mais revient, quelques jours après la mise à l'herbe, à sa valeur initiale.

\section{INTRODUCTION}

Le but du présent travail est d'étudier les variations de composition de la matière grasse du beurre provoquées par la mise à l'herbe du troupeau. La mise à l'herbe désigne le passage, à une date déterminée, du régime de stabulation au régime de pâturage. Ainsi qu'en témoignent les travaux de divers auteurs, c'est une pratique générale dans les pays scandinaves (BERGMAN et JoOST, I953 ; SWARTIING et MATTSSON, I956 ; STEEN, I956), en Hollande (STADHOUdERS et MUldER, I955), en Allemagne (Schwar'Tz, LUDWIG et GIESSLER, I957), en Belgique (JAMOTTE et Piraux I957) et en France, du moins dans l'Est (BEJAmbes et SAvore, I954), le Centre-Ouest (KUzDzal,-Savole, I959 $a$ ) et le Bassin-Parisien (Kuzdzal-Savore, I959 b), c'est-àdire dans la majorité des régions de grande production laitière. 
La lecture de travaux poursuivie dans d'autres pays montre qu'en NouvelleZélande (Cox et MacDowali, I948; HANsen et ShorLand, I952) et dans certaines régions des États-Unis (ZEHREN et JACKSON, I956), du Canada (Wood, I956 ; Wood et $\mathrm{HAAB}_{\mathrm{A}}$ I957) ou même de France (différentes parties de la Normandie (BEJAMBES et SAvorE, I954), les vaches laitières restent au pâturage toute l'année et y reçoivent pendant la saison froide un complément alimentaire destiné à pallier le manque d'herbe.

Dans les pays où alternent le régime de stabulation et le régime de pâturage, on observe une grande amplitude de variation des indices de la matière grasse du beurre, de brusques modifications lors de la mise à l'herbe ou lors de la rentrée à l'étable du troupeau et l'apparition à des époques fixes et identiques pour les différents pays, de maxima et de minima des divers indices.

Au contraire, dans les pays pour lesquels la période de pâturage s'étend sur toute l'année, l'amplitude des variations est beaucoup plus faible et les maxima et minima se situent à des périodes variables.

A Jouy-en-Josas existe l'alternance du régime de stabulation et du régime de pâturage : aussi nous n'examinerons dans ce qui va suivre que les résultats tirés d'expériences poursuivies dans des conditions semblables à celles devant lesquelles nous étions nous-mêmes placés.

TABLEAU I

Proportions des acides polyinsaturés dans la matière grasse du lait (en $g$ pour $100 \mathrm{~g}$ de matière grasse).

\begin{tabular}{|c|c|c|c|c|c|c|c|}
\hline \multirow[b]{2}{*}{ Source } & \multirow[b]{2}{*}{ Date de prélèvement } & \multicolumn{3}{|c|}{ Conjugués } & \multicolumn{3}{|c|}{ Non conjugués } \\
\hline & & Diènes & Triènes & Tétraènes & $\begin{array}{l}\text { Diènes } \\
\text { (lino- } \\
\text { lénique) }\end{array}$ & $\begin{array}{c}\text { Triènes } \\
\text { (lino- } \\
\text { lénique) }\end{array}$ & Tétraènes \\
\hline $\begin{array}{l}\text { StadHouders } \\
\text { et MulDER } \\
(1955)\end{array}$ & $\begin{array}{l}27-4-54 \\
11-5-54 \\
25-5-54\end{array}$ & $\begin{array}{l}0,81 \\
1,72 \\
2,10\end{array}$ & $\begin{array}{l}0,020 \\
0,023 \\
0,023\end{array}$ & $\begin{array}{l}0,003 \\
0,004 \\
0,003\end{array}$ & $\begin{array}{l}1,57 \\
0,73 \\
0,86\end{array}$ & $\begin{array}{l}0,46 \\
0,81 \\
0,84_{4}\end{array}$ & $\begin{array}{l}0,37 \\
0,27 \\
0,42\end{array}$ \\
\hline $\begin{array}{c}\text { PEDERSEN et } \\
\text { OVERGAARD } \\
(1958)\end{array}$ & $\begin{array}{l}\text { Race } R D M \\
\text { avril } \\
\text { mai } \\
\text { juin } \\
\text { Race Jerseyaise } \\
\begin{array}{l}\text { avril } \\
\text { mai } \\
\text { juin }\end{array}\end{array}$ & & $\begin{array}{c}\text { onjugués } \\
0,58 \\
0,60 \\
1,35 \\
\\
0,44 \\
0,67 \\
0,86\end{array}$ & $\mathrm{C} 18$ & $\begin{array}{c}0,53 \\
0,75 \\
0,46 \\
\\
0,27 \\
0,61 \\
0,43\end{array}$ & $\begin{array}{l}0,55 \\
0,57 \\
0,66 \\
\\
0,38 \\
0,92 \\
0,60\end{array}$ & $\begin{array}{l}- \\
- \\
- \\
-\end{array}$ \\
\hline $\begin{array}{l}\text { SWARTLING } \\
\text { et MATTSSON } \\
(1956)\end{array}$ & $\begin{array}{c}\text { Race SLB } \\
29-\int_{\mathrm{t}}-53 \\
27-5-53 \\
17-6-53 \\
\text { Race Jerseyaise } \\
29-4_{4}-53 \\
27-5-53 \\
17-6-53\end{array}$ & & $\begin{array}{l}0,48 \\
2,17 \\
2,52 \\
\\
0,48 \\
1,71 \\
1,92\end{array}$ & . & $\begin{array}{l}1,23 \\
1,33 \\
0,21 \\
\\
1,18 \\
0,80 \\
0,23\end{array}$ & $\begin{array}{l}0,90 \\
1,17 \\
1,06 \\
\\
0,97 \\
1,08 \\
1,15\end{array}$ & $\begin{array}{l}- \\
- \\
- \\
-\end{array}$ \\
\hline
\end{tabular}


Les dates de fabrication des échantillons de beurre analysés au cours de ces diverses expériences sont généralement espacées d'une quinzaine de jours. On connaît donc seulement le résultat global de la mise à l'herbe sur la composition de la matière grasse du beurre.

En ce qui concerne les indices on note une augmentation très nette de l'indice d'iode et une diminution de l'indice de saponification et de l'acidité volatile. Nous avons montré récemment (KUZDZAL-SAvorE, I959 b) que ces mođifications apparaissaient dans les 48 heures qui suivent la mise à 1'herbe.

En ce qui concerne les acides gras, STADHOUDERs et MuLDER (I956) notent, entre le 30 mars et le 2 mai 1954 , une diminution de la teneur en acides myristique et palmitique respectivement 13,6 à II,4 et 28,6 à 22 , I moles p. Ioo moles d'acides totaux. Euntre ces mêmes dates les teneurs en acides stéarique et oléique s'élèvent respectivement de 8,2 à I0,5 et de 23,7 à 3I,0 moles p. Ioo tnoles d'acides totaux.

Nous avons rassemblé dans le tableau $\mathrm{I}$, les résultats concernant les acides polyinsaturés, rapportés par différents auteurs. On constate que la mise à l'herbe des vache laitières entraîne une augmentation nette du pourcentage des acides diènes conjugués, une augmentation légère du pourcentage des acides triènes non conjugués (acide linolénique) et des résultats variables en ce qui concerne les acides diènes non conjugués (acide linoléique). Pour ces derniers, S'TADHOuders et MULDER (I955) observent une nette diminution suivie aussitôt d'une légère augmentation. Inversement, et pour ce même groupe d'acides, JENSEN, PEDERSEN et OVERGAARD (I958) notent, d'avril à juin, une augmentation suivie d'une diminution. Enfin, Swarting et MaTTsson (I956) relèvent peu de variation du contenu en acide linoléique entre avril et mai, mais signalent en juin des valeurs faibles (o,2 g p. Ioo g de matière grasse).

LEMBKE et KAUFMANN (I954) ont noté quelques pourcentages d'acides triènes conjugués de laits individuels provenant de vaches récemment conduites au pâturage. Au cours des douze premiers jours de pâturage l'augmentation est de 0,05 à $0,09 \mathrm{~g}$ p. Ioo g de matière grasse. Ces chiffres sont élevés par rapport aux valeurs signalées pat STADHOUdERs et MulDER (I955), qui sont voisines de 0,020.

Compte-tenu des résultats précédents, il a semblé utile d'étudier la composition de la matière grasse du beurre fabriqué à partir du lait de mélange produit chaque jour et à chaque traite par les vaches laitières appartenant à un même troupeau et soumises à des conditions de vie identiques et ceci pendant une période d'environ deux semaines encadrant la mise à l'herbe des vaches laitières.

Cette étude a été poursuivie au cours de trois printemps successifs : I957, I958 et 1959 .

\section{MATÉRIEL, ET MÉTHODES}

a) Modalité de la mise à l'herbe.

Ces modalités sont décrites sur la figure I.

Le passage brusque du régime de stabulation au régime de pâturage a existé pour les groupes I ( 7 vaches) et III ( 40 vaches). On distribua cependant un peu de foin et de paille d'avoine aux vaches du groupe I une fois en pâture et, pendant les trois jours suivant la mise à l'herbe, les vaches du groupe III rentrèrent la nuit à l'étable où elles reçurent du foin et de l'ensilage, mais, dans un cas comme dans l'autre, la consommation de ces aliments, notable le lendemain de la mise à l'herbe, fut presque nulle les jours suivants.

Le passage ménagé du régime de stabulation au régime de pâturage a existé :

- pour le groupe II dont les 22 vaches reçurent une ration d'herbe fraîche à l'étable en rem- 
placement d'une fraction de la ration de betterave, pendant une période de 8 jours précédant la sortie au pâturage.
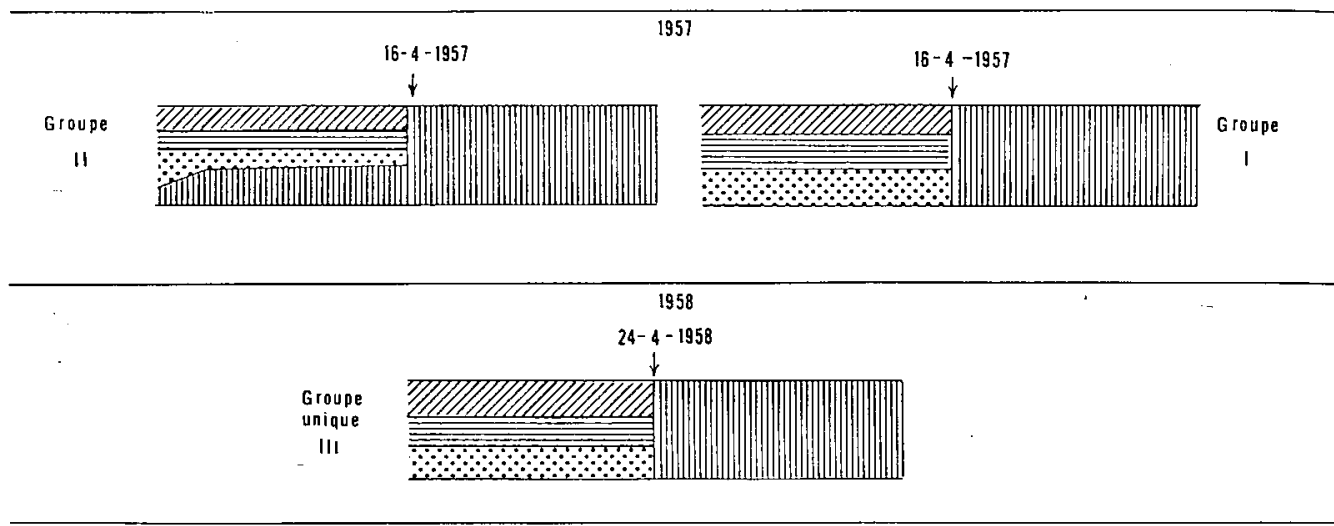

1959
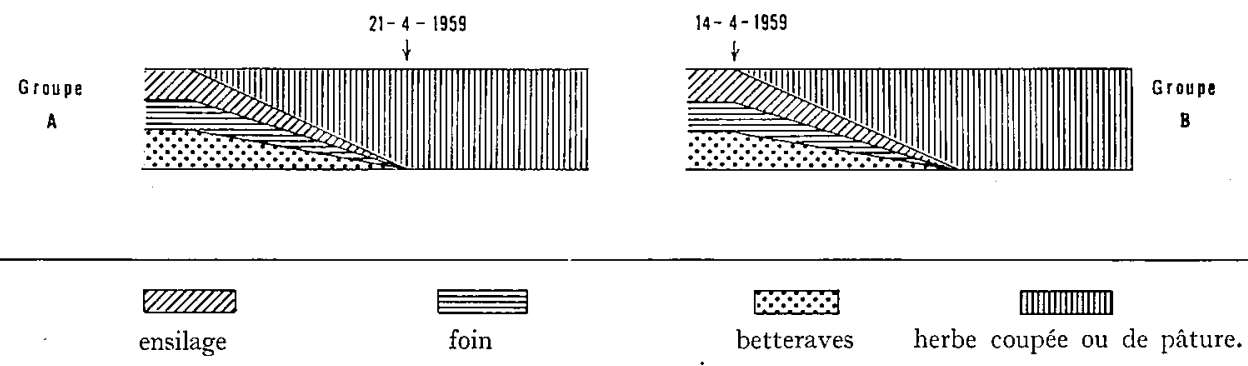

FIG. I - Modalités de la mise à l'herbe : la Aèche indique la sortie au pâturage.

- pour les groupes A et B. Du I4 au 2 I avril I959, les I2 vaches du groupe B restèrent au pâturage un temps chaque jour plus long et les I2 vaches du groupe A restèrent à l'étable et reçurent une quantité d'herbe fraîche chaque jour un peu plus grande. Pendant le même temps (I5 au $2 \mathrm{r}$ avril) les rations de foin, d'ensilage et de betteraves des vaches des groupes $\mathrm{A}$ et $\mathrm{B}$ diminuèrent progressivement.

\section{b) Prélèvements du lait.}

Les prélèvements eurent lieu lors de la traite du matin et lors de la traite du soir, du 8 au 25 avril en I957, du 21 au 30 avril en $195^{8}$ et du I3 au 27 avril en I959.

On prélève des échantillons sur le lait provenant de la traite de chacune des vaches retenues pour l'expérimentation. Les échantillons correspondant aux vaches d'un même groupe sont pondérés, puis réunis pour former un échantillon moyen d'un volume total de 4 à 5 litres, représentatif de la traite.

\section{c) Préparation de l'échantillon de beurre.}

Les laits de mélange sont écrémés. La crème est placée I à 2 heures à la glacière, puis transformée en beurre au moyen d'un "mixer " tournant à I2.00o tours par minute. Le beurre est ensuite lavé, malaxé au moyen de palettes en bois et empaqueté dans du papier sulfurisé. Les échantillons sont entreposés à $-{ }_{1}^{\circ}{ }^{\circ}$.

d) Analyses.

Le beurre est fondu au bain-marie à $70^{\circ} \mathrm{C}$ et centrifugé. L'analyse est effectuée sur la matière grasse surnageante; elle comporte la détermination de divers indices et le closage des acides polyinsaturés. 
Les échantillons de beurre préparés en avril I 958 ont été analysés en février, mars et avril I959. Les échantillons de beurre préparés en avril I957 et avril r959 ont été analysés dès la fin des prélèvements, c'est-à-dire, respectivement, en mai et juin 1957 , en mai, juin et juillet I959.

r. Indices. - On a déterminé l'indice d'iode (selon WIJs), l'indice de saponification, l'acidité volatile soluble et insoluble. La détermination de l'acidité volatile a été effectuée selon le procédé officiel du Service de la Répression des Fraudes.

2. Acides poly-insaturés. - Les acides poly-insaturés ont été dosés (sur les échantillons de I958 et de 1959) par spectrophotométrie dans l'ultra-violet suivant la méthode décrite par BRICE et SWain (I945). On a utilisé pour la lecture de l'absorption un spectrophotomètre BECKMAN, D. U.

Le dosage des acides poly-insaturés comportant des doubles liaisons en position conjuguée est effectué sur la matière grasse dissoute dans le cyclohexane. Les acides polyinsaturés comportant des doubles liaisons en position non conjuguée sont dosés après isomérisation alcaline. On a utilisé, pour effectuer l'isomérisation alcaline, une solution de potasse dans le glycérol à $I 7,5$ p. I $00(p / v)$. Après isomérisation la solution est diluée avec de l'alcool éthylique absolu.

Les proportions des divers acides poly-insaturés ont été obtenues en suivant la méthode de calcul indiquée par BRICE et SWAIN (1945).

\section{RÉSULTA.TS}

Nous étudierons successivement les résultats concernant les indices de la matière grasse et ceux relatifs au dosage des acides poly-insaturés.

\section{A. - VARIATIONS DES INDICES DE LA MATIÈRE GRASSE}

\section{I. - Passage brusque du régime de stabulation au régime de pâturage (figure 2).}

Les variations observées en I957 et I958 suivent une évolution parallèle en ce qui concerne l'indice d'iode, l'indice de saponification et l'acidité volatile insoluble mais présentent une évolution différente en ce qui concerne l'acidité volatile soluble.

La mise à l'herbe ayant lieu le jour J à I5 heures, l'augmentation de l'indice d'iode est déjà notable moins de $I 6$ heures après la sortie des vaches. Cette augmentation s'amplifie au cours des jours suivants et, en 48 heures, du soir du jour J au soir du jour $\mathrm{J}+2$, l'indice d'iode augmente de I 2 unités en 1957 et de II,5 unités en 1958 . Pendant ces 48 heures l'indice d'iode du matin est supérieur puis égal à l'indice d'iode du soir précédent, alors qu'en régime d'alimentation uniforme, l'indice d'iode du matin reste généralement inférieur à l'indice d'iode du soir (précédent ou suivant). L'indice d'iode maximum est atteint 4 jours après la mise à l'herbe ; on observe ensuite une légère diminution.

Après la mise à l'herbe des vaches laitières, l'indice de saponification diminue mais avec un retard de $\mathrm{I} 2$ heures sur le moment oì l'indice d'iode commence à augmenter. Du soir du jour J au soir du jour J + 4, on observe en I957 comme en I958 une diminution de I2 unités.

Les variations de l'acidité volatile insoluble présentent un aspect très voisin de celles de l'indice de saponification. Du soir du jour $\mathrm{J}$ au soir du jour $\mathrm{J}+4$, la diminution est de 4,5 à 3,0 en I 957 et de 4,0 à 2 , I en 1958 .

L'amplitude de variation de l'acidité volatile soluble entre le soir du jour $\mathrm{J}$ et le soir du jour J + 4 est, aussi bien en I957 qu'en I958, voisine de 4 unités mais l'allure des courbes diffère d'une année à l'autre.

Ainsi lorsque le passage du régime de stabulation au régime de pâturage se fait brusquement, on peut dire qu'à peu de chose près, les valeurs les plus élevées pour l'indice d'iode et les valeurs les plus faibles pour l'indice saponification et l'acidité volatile soluble et insoluble se rencontrent dans la matière grasse du beurre fabriqué à partir du lait produit le soir du jour $\mathrm{J}+4$. 
FIg. 2 - Passage brusque du régime de stabulation au régime de pälurage. Variation des indices de la matière grasse du beurre.

-— I957 (groupe II) A - - 1958.

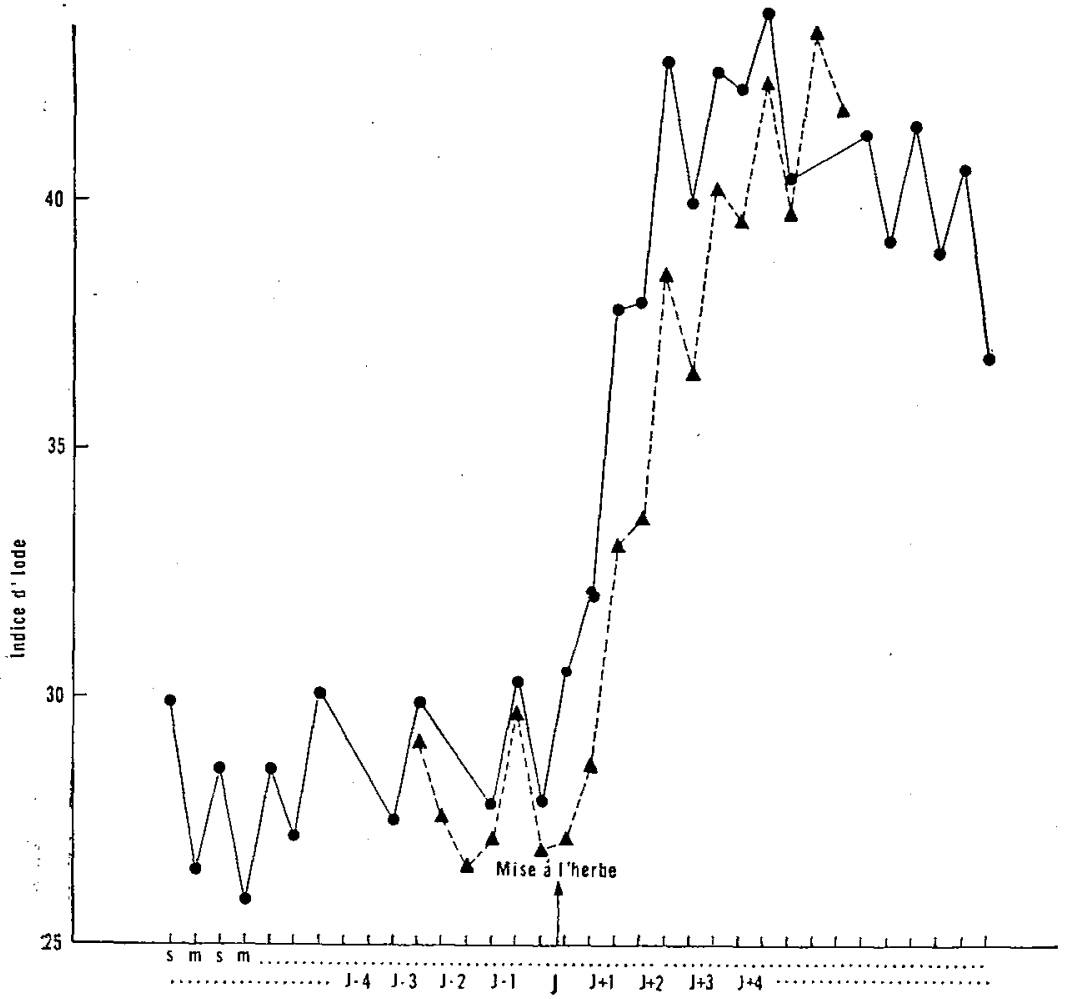

a) Indice d'iode.

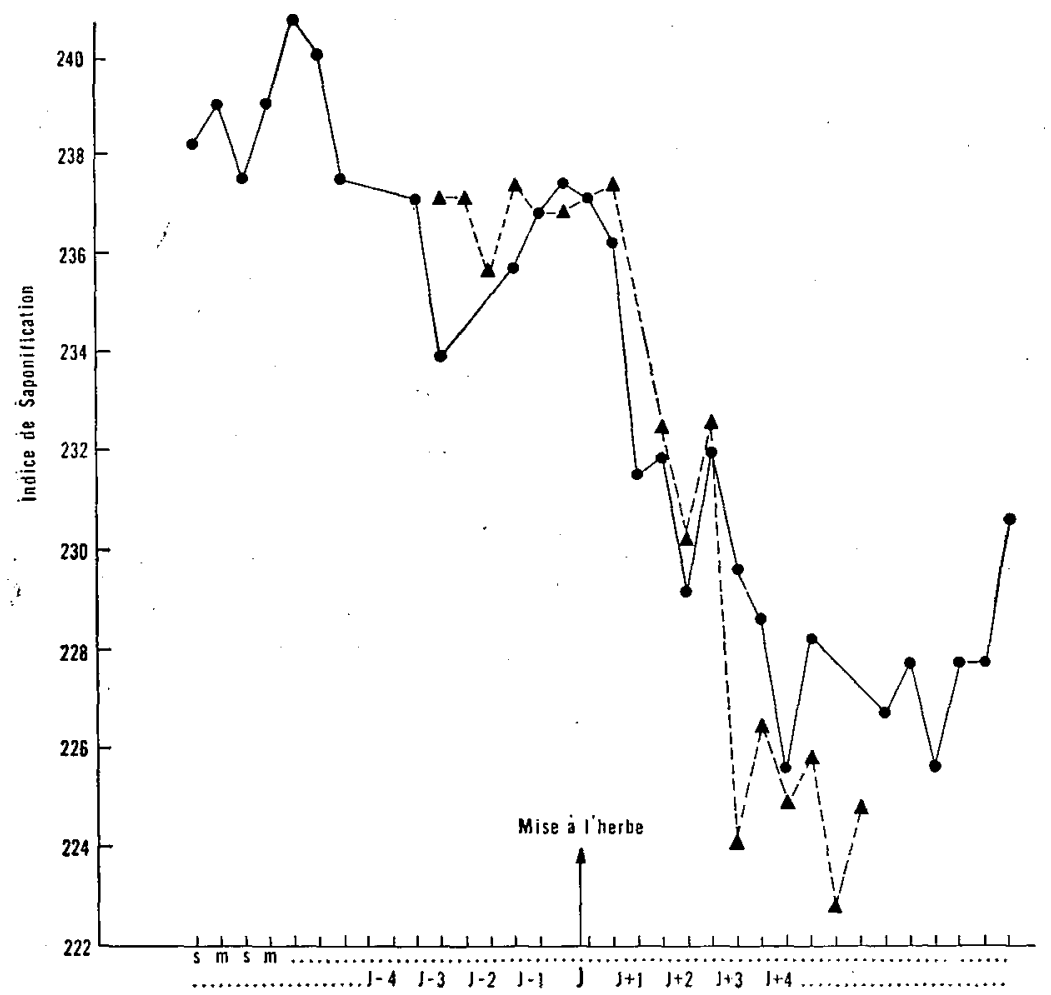

b) Indice de saponification. 

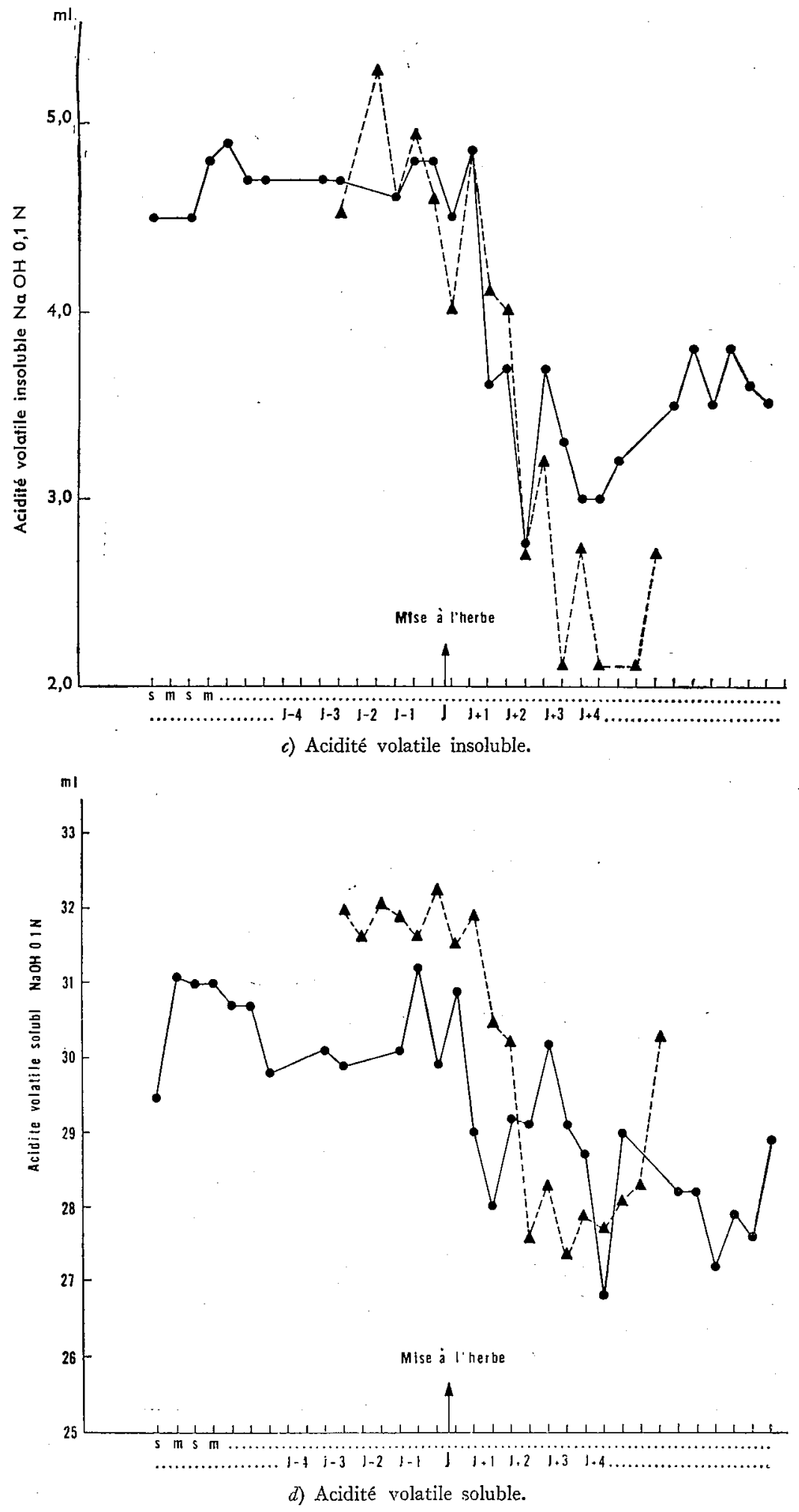


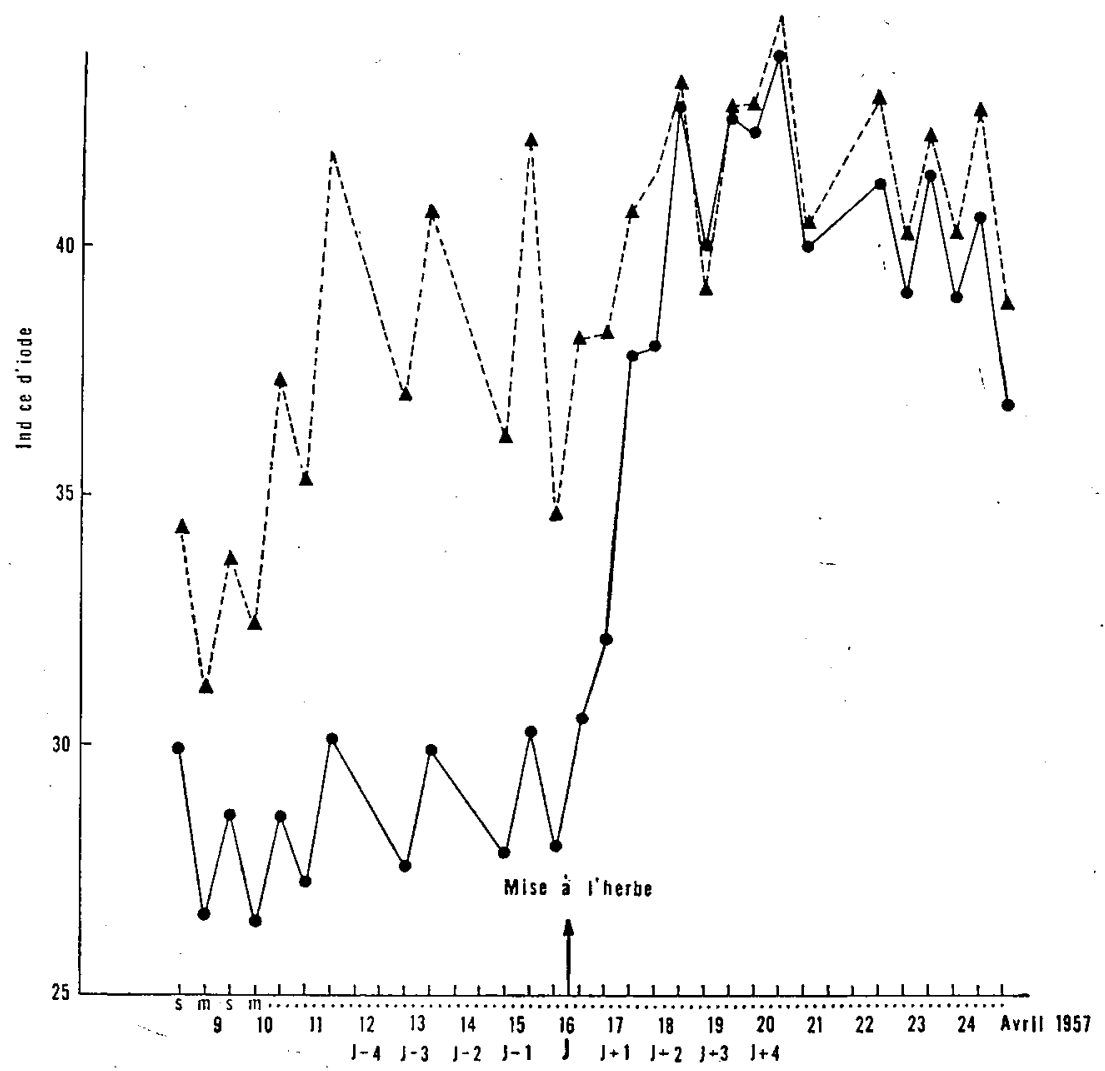

a) Indice d'iode.

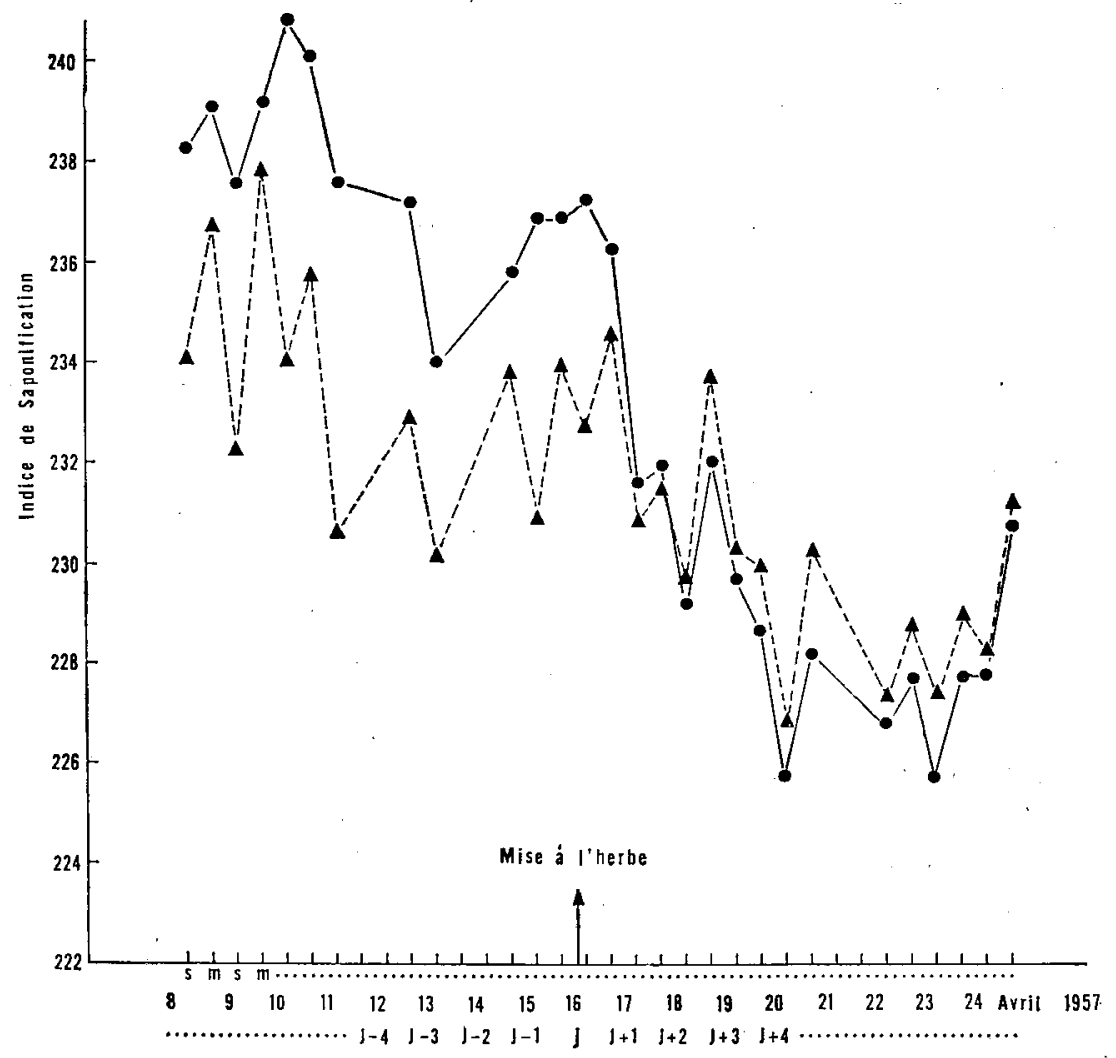

b) Indice de saponification. 
FIg. 3 - Inffuence d'une distribution d'herbe à l'étable (1957). Variation des indices de la matière grasse Les vaches du groupe I (
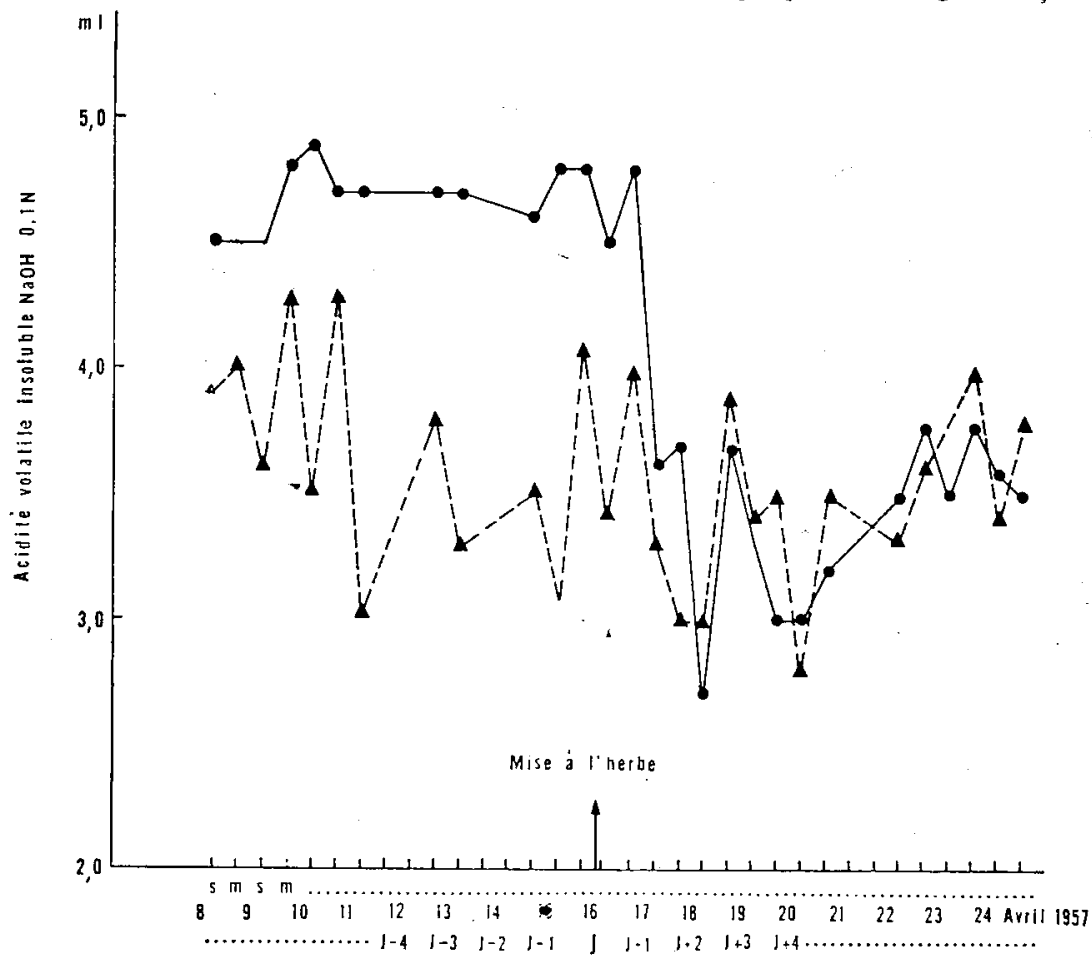

c) Acidité volatile insolubie.

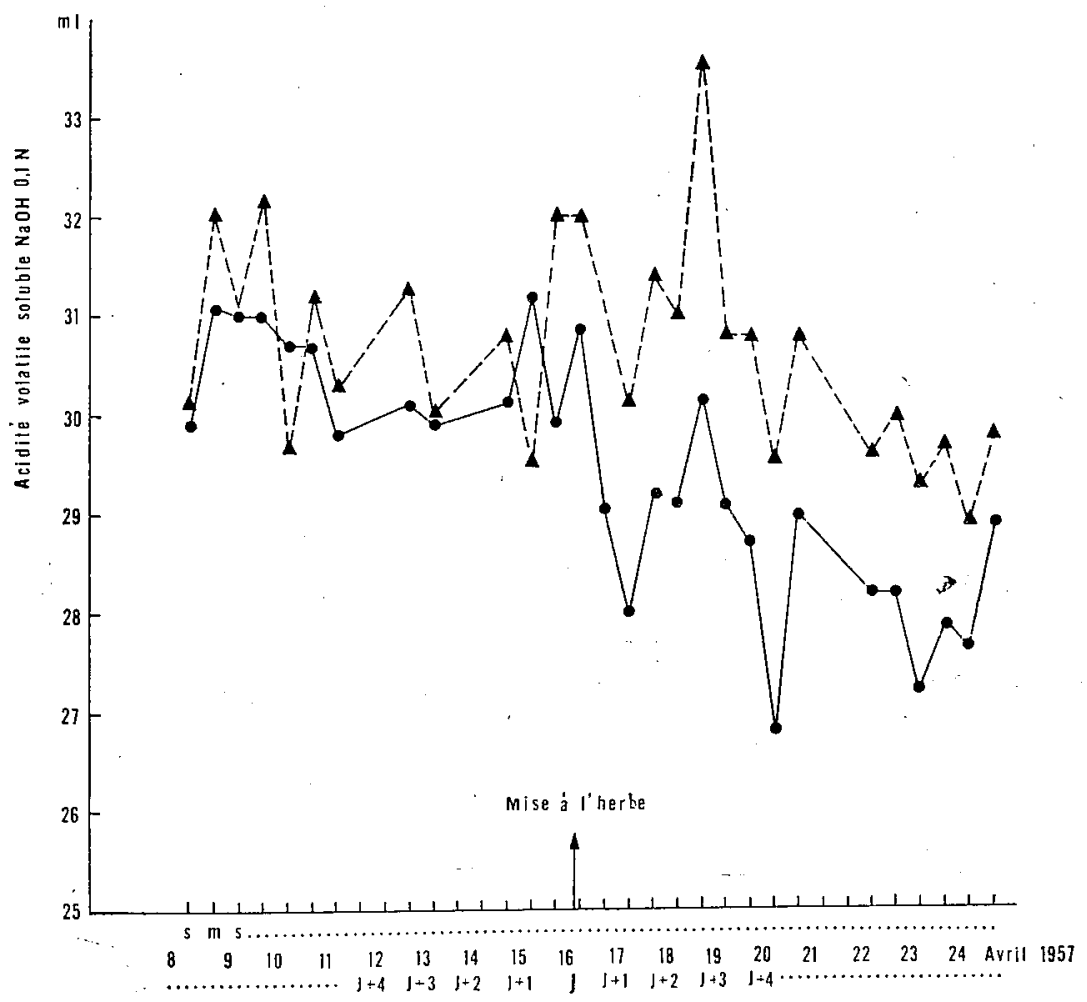

d) Acidité volatile soluble. 
2. - Intluence de la distribution d'herbe à l'étable (figure 3).

Au début de l'expérience un écart existe entre les indices d'iode, les indices de saponification et les acidités volatiles insolubles de chacun des deux groupes.

La distribution d'herbe fraîche accentue cet écart sauf en ce qui concerne 1'indice de saponification. Les acidités volatiles solubles, par contre, restent équivalentes pour les deux groupes, au début comme au cours de l'expérimentation.

Cette distribution d'herbe fait apparaître de très nettes différences entre la composition de la matière grasse du matin et celle du soir. L'indice d'iode du soir est supérieur à l'indice d'iode du matin de près de 6 tunités tandis que l'indice de saponification et l'acidité volatile sont régulièrement plus élevés le matin que le soir.

3. - Passage ménagé du régime de stabulation au régime de pâturage (fig. 4).

L'indice d'iode a seul été étudié.

A partir du jour où l'herbe fraîche est introduite dans l'alimentation des vaches laitières, on assiste à une augmentation progressive de l'indice d'iode, augmentation

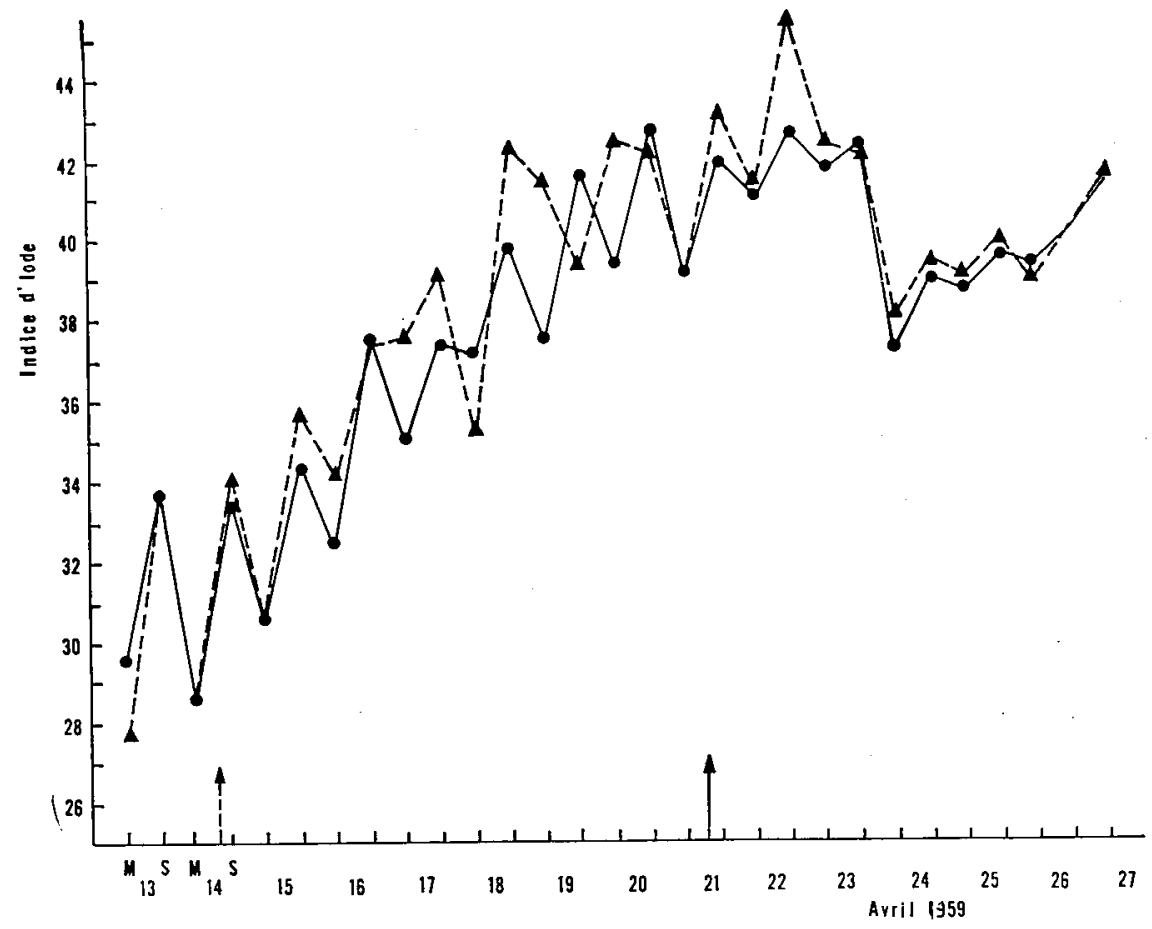

Fig. 4 - Passage ménagé du régime de stabulation au régine de pâturage (1959)). Variation de l'indice d'iode.

troupeau A $(\mathbf{O}-\mathbf{O})$ troupeau $\mathrm{B}(\boldsymbol{\Delta}---\mathbf{\Delta})$.

en quelque sorte " contenue ». En effet, d'une part l'indice d'iode du matin reste généralement (et même, toujours pour le groupe A) inférieur à l'indice d'iode du soir précédent comme cela semble être de règle en régime stable et, d'autre part, l'indice d'iode le plus élevé est atteint 24 heures après l'établissement du régime de pâturage 
complet, alors qu'il était atteint seulement le $4^{\mathrm{e}}$ jour dans le cas du changement brusque de régime alimentaire.

L'amplitude totale de variation del'indice d'iode (calculée d'un matin à un autre) est de I3 unités en moyenne. En fait, du I3 au 23 avril, l'augmentation de l'indice d'iode pour le groupe $B$ sorti aut pâturage dès le $I_{4}$ avril dépasse de 3 unités l'augmentation de l'indice d'iode se rapportant au groupe A sorti seulement le $2 \mathrm{I}$ avril. L'environnement " plein air » semble ainsi avoir amplifiél'influence spécifique de l'alimentation à l'herbe.

\section{B. 一 ÉTUDE DES ACIDES POLYINSATURÉS}

I. - Passage brusque du régime de stabulation au régime de pâturage (I958).

Le tableau 2 et la figure 5 donnent les valeurs del'indice d'iode et les proportions des différents acides poly-insaturés de la matière grasse du beurre provenant de l'ensemble

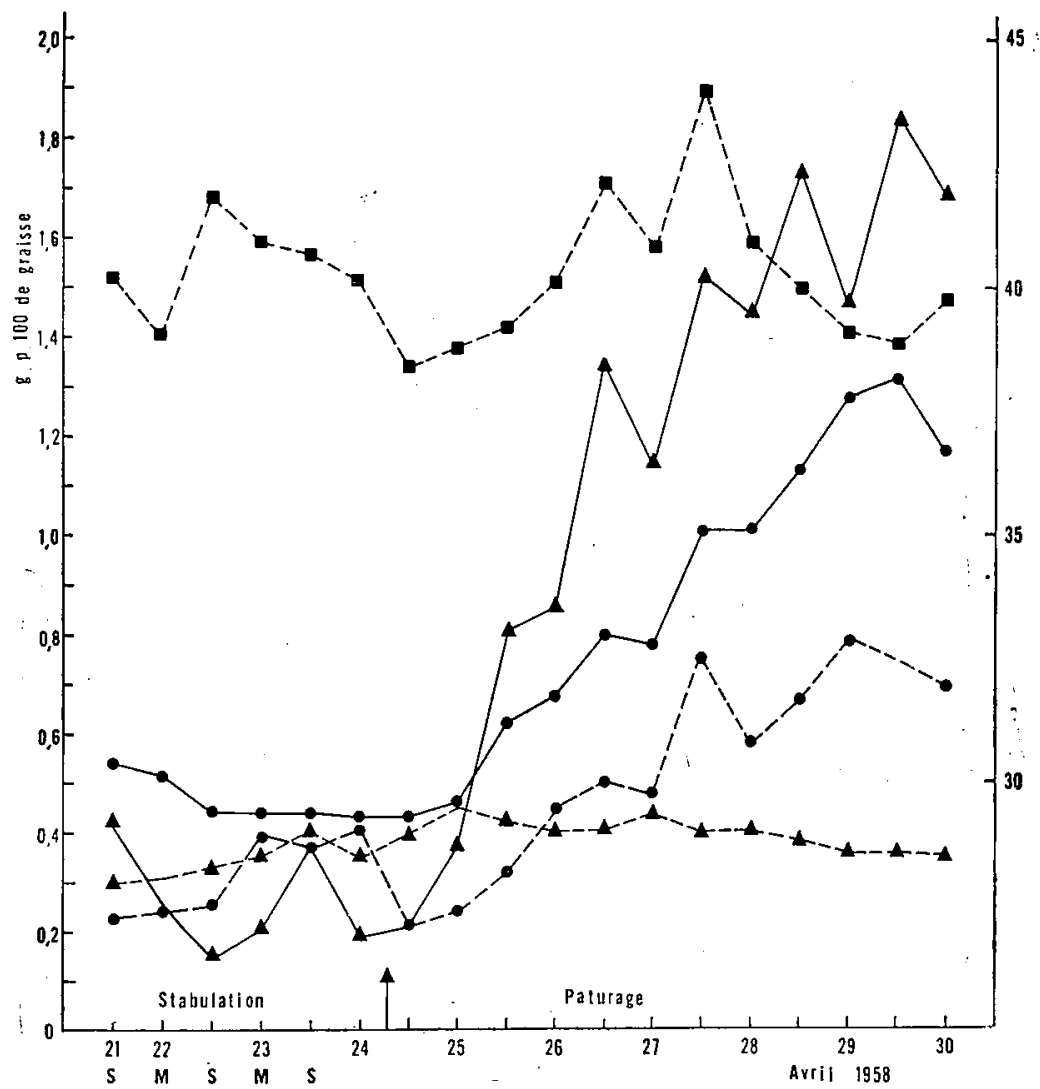

FIG. 5 - Passage brusque du régime de stabulation au régime de pâturage.

Variation de l'indice d'iode ( de diènes (

non conjugués de la matière grasse du beurre.

des vaches laitières du 2I au 30 avril I958. Dans le tableau 3 on porte les proportions moyennes des différents acides polyinsaturés, avant ( 2 I au 25 avril matin) et après (25 avril matin au 30 avril) la mise à 1 'herbe. 


\section{TABLEAU 2}

Passage brusque du régime de stabulation au régime de pâturage. (Mise à l'herbe : 24 avril 1958).

Variations de l'indice d'iode et des proportions des acides polyinsaturés de la matière grasse du lait.

(en $\mathrm{g}$ pour $100 \mathrm{~g}$ de matière grasse).

\begin{tabular}{|c|c|c|c|c|c|}
\hline \multirow{3}{*}{$\begin{array}{l}\text { Dates des } \\
\text { prélèvements }\end{array}$} & \multirow{3}{*}{ Indice d'iode } & \multicolumn{4}{|c|}{ Acides polyinsaturés } \\
\hline & & \multirow{2}{*}{$\frac{\text { Conjugués }}{\text { Diènes }}$} & \multicolumn{3}{|c|}{ Non conjugués } \\
\hline & & & Diènes & Triènes & Tétraènes \\
\hline $21-4$ soir $\ldots \ldots \ldots \ldots$ & 29,1 & 0,5 & 1,5 & 0,2 & 0,3 \\
\hline $22-4$ matin $\ldots \ldots \ldots$ & 27,6 & 0,5 & 1,4 & $0, \underline{2}$ & 0,3 \\
\hline $22-4$ soir. & 26,5 & 0,4 & 1,7 & 0,3 & 0,3 \\
\hline $23-4$ matin .. & 27,1 & 0,4 & 1,6 & $0,{ }^{\prime}$ & $0, l_{4}$ \\
\hline $23-4$ soir $\ldots \ldots \ldots \ldots$ & 28,7 & 0,4 & 1,6 & $0,{ }_{4}$ & 0,4 \\
\hline 24-4 matin $\ldots \ldots \ldots$ & 26,9 & 0,4 & 1,5 & 0,4 & 0,4 \\
\hline $24-4$ soir $\ldots \ldots \ldots \ldots$ & 27,1 & 0,4 & 1,3 & 0,2 & 0,4 \\
\hline $25-4$ matin $\ldots \ldots \ldots$. & 28,7 & 0,5 & 1,4 & 0,2 & 0,5 \\
\hline $25 \cdot 4$ soir $\ldots \ldots \ldots \ldots$ & 33,0 & 0,6 & $1, \mathbf{l}_{\mathrm{k}}$ & 0,3 & 0,4 \\
\hline $26-1$ matin $\ldots \ldots \ldots \ldots$ & 33,6 & 0,7 & 1,5 & 0,5 & 0,4 \\
\hline $26-t_{1}$ soir $\ldots \ldots \ldots \ldots$ & 38,4 & 0,8 & 1,7 & 0,5 & 0,4 \\
\hline $27-4$ matin $\ldots \ldots \ldots$ & 36,4 & 0,8 & 1,6 & 0,5 & 0,4 \\
\hline $27-4$ soir $\ldots \ldots \ldots \ldots$ & 40,3 & 1,0 & 1,9 & 0,8 & 0,4 \\
\hline 28-4 matin $\ldots \ldots \ldots$ & 39,5 & 1,0 & 1,6 & 0,6 & 0,4 \\
\hline $28-4$ soir $\ldots \ldots \ldots \ldots$ & 42,4 & 1,1 & 1,5 & 0,7 & 0,4 \\
\hline 29-4 matin . . . . . . & 39,7 & 1,3 & 1,4 & 0,8 & 0,4 \\
\hline $29-4$ soir $\ldots \ldots \ldots$ & 43,5 & 1,3 & $1,4_{4}$ & 0,8 & 0,4 \\
\hline $30-4$ matin $\ldots \ldots \ldots$ & 41,8 & 1,2 & 1,5 & 0,7 & 0,4 \\
\hline
\end{tabular}

On voit que la mise à l'herbe des vaches laitières provoque une augmentation très nette des acides diènes conjugués (1a proportion de diènes conjugués trouvée le 29 avril est approximativement triple de la proportion trouvée le 2I avril) et une augmentation sensible (environ deux fois la teneur initiale) des triènes non conjugués. Par contre on retrouve en fin d'expérimentation (le 30 avril) une proportion d'acide linolénique $(\mathrm{I}, 5)$ égale à la proportion trouvée au début (le $2 \mathrm{I}$ avrii). La proportion d'acide arachidonique est également stable.

\section{TABLEAU 3}

Proportions moyennes des différents acides polyinsaturés.

(En g pour roo $g$ de matière grasse).

\begin{tabular}{|c|c|c|}
\hline & Du 21 au 25 avril & Du 25 au 30 avril \\
\hline Diènes conjugués & 0,46 & 0,98 \\
\hline Acide linoléique. & 1,49 & 1,55 \\
\hline Acide linolénique . & 0,29 & 0,60 \\
\hline Acide arachidonique & 0,37 & 0,39 \\
\hline
\end{tabular}




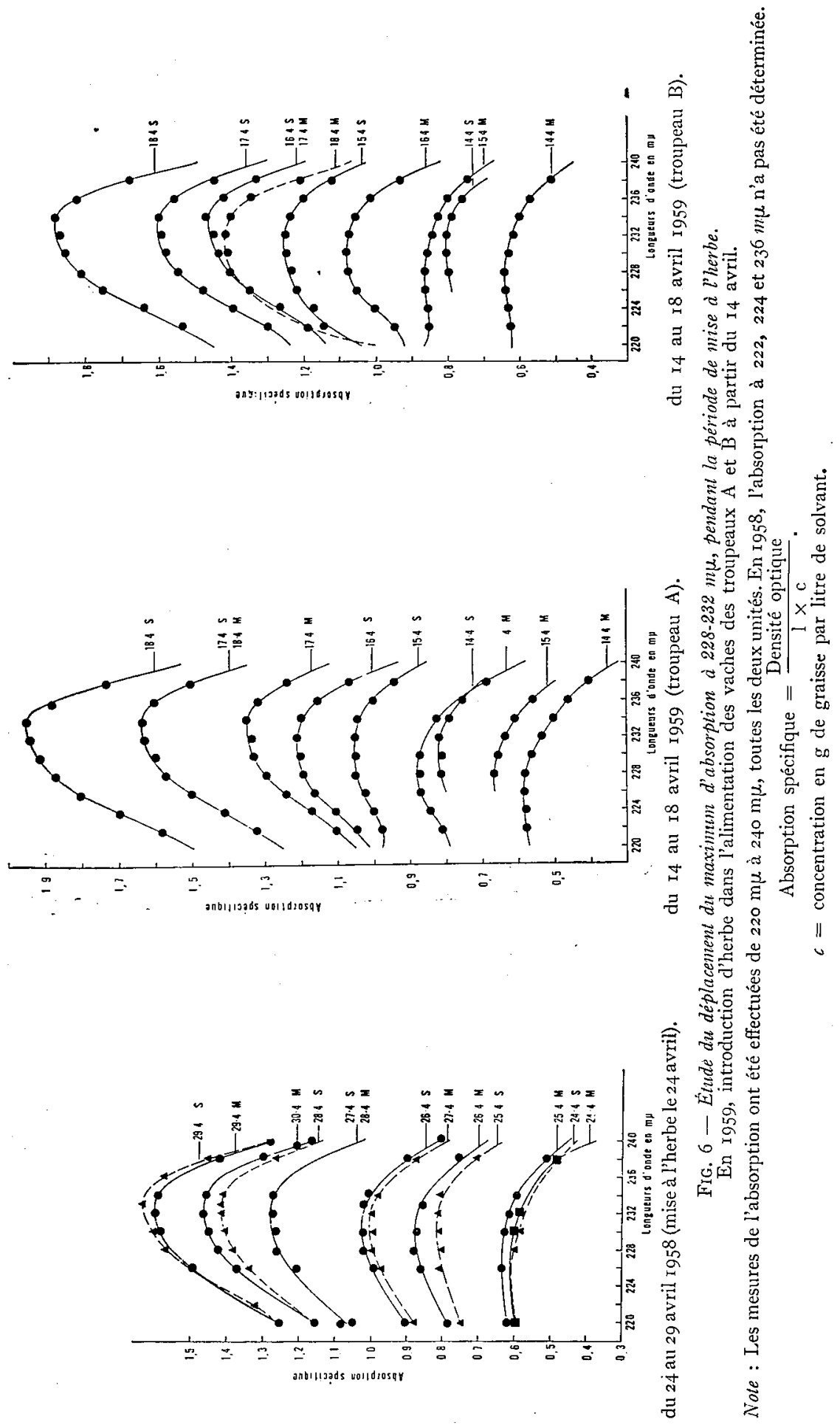


Du 25 au 28 avril I958 le sens des variations de l'indice d'iode et des proportions d'acides diènes conjugués, diènes et triènes non conjugués est le même. A partir du 28 avril ce parallélisme n'est plus observé : en effet, la proportion d'acide linoléique s'abaisse alors que s'élèvent encore les proportions d'acides diènes conjugués et triènes non conjugués.

Sur la matière grasse non isomérisée provenant des échantillons prélevés en I958, un maximum d'absorption est régulièrement observé à $268 \mathrm{~m} \mu$ et seulement une inflexion de la courbe à $3 \mathrm{r} 6 \mathrm{~m} \mu$. Si l'on considère que l'absorption à ces longueurs d'onde est due à la présence d'acides triènes et tétraènes conjugués, on trouve, en suivant la méthode de calcul indiquée par BRICE et SwAIN (I945), pour les acides triènes conjugués 0,010 à 0,019 (moyenne 0,0128 ) $g$ pour IOO $g$ de matière grasse et pour les acides tétraènes conjugués 0,001 à $0,002 \mathrm{~g}$ pour roo $\mathrm{g}$ de matière grasse.

Observation concernant l'absorption à 232 mu. - D'après les auteurs le maximum d'absorption correspondant aux diènes conjugués se place à 232-233 m $\mu$. Nous con-

\section{TABLEAU 4}

Passage ménagé du régime de stabulation au régime de pâturage. Troupeau $B$. Variations de l'indice d'iode et des proportions des acides polyinsaturés de la matière grasse du lait.

\begin{tabular}{|c|c|c|c|c|c|}
\hline \multirow{3}{*}{$\begin{array}{c}\text { Dates } \\
\text { des } \\
\text { prélèvements }\end{array}$} & \multirow{3}{*}{ Indice d'iode } & \multicolumn{4}{|c|}{ Acides polyinsaturés (en g pour $100 \mathrm{~g}$ de matière grasse) } \\
\hline & & \multirow{2}{*}{$\begin{array}{c}\text { Conjugués } \\
\text { Diènes }\end{array}$} & \multicolumn{3}{|c|}{ Non conjugués } \\
\hline & & & Diènes & Triènes & Tétraènes \\
\hline $13-4$ matin $\ldots \ldots \ldots$ & 27,6 & 0,5 & 1,0 & 0,4 & 0,4 \\
\hline $13-4$ soir $\ldots \ldots \ldots \ldots$ & 33,7 & 0,6 & 1,2 & 0,5 & 0,4 \\
\hline $14-4_{4}$ matin $\ldots \ldots \ldots$ & 28,5 & 0,5 & 1,1 & 0,5 & 0,4 \\
\hline $14-4$ soir $\ldots \ldots \ldots \ldots$ & $34,4_{x}$ & 0,6 & 1,4 & 0,5 & 0,3 \\
\hline $15-4$ matin $\ldots \ldots \ldots$ & 30,9 & 0,7 & 0,9 & $0,,^{\prime}$ & 0,4 \\
\hline $15-4$ soir $\ldots \ldots \ldots$ & 35,8 & 1,0 & 1,0 & 0,5 & 0,4 \\
\hline $16-4^{\prime}$ matin $\ldots \ldots \ldots$ & 34,4 & 0,9 & 1,0 & 0,5 & 0,4 \\
\hline $16-4$ soir $\ldots \ldots \ldots \ldots$ & 37,5 & 1,2 & 1,3 & $0, f_{t}$ & 0,4 \\
\hline $17-4$ matin $\ldots \ldots \ldots$ & 37,7 & 1,2 & 1,5 & 0,5 & 0,4 \\
\hline $17-4$ soir $\ldots \ldots \ldots$ & 39,2 & 1,3 & $1, l_{t}$ & 0,6 & 0,4 \\
\hline $18-4$ matin $\ldots \ldots \ldots$ & 35,1 & 1,1 & 1,1 & 0,5 & 0,3 \\
\hline $18-4$ soir $\ldots \ldots \ldots$ & 42,3 & 1,5 & 1,4 & 0,5 & 0,3 \\
\hline $19-4$ matin $\ldots \ldots \ldots$ & 41,3 & 1,4 & $1, c_{4}$ & 0,4 & 0,3 \\
\hline $19-4$ soir $\ldots \ldots \ldots$ & 39,3 & 1,5 & 1,5 & 0,2 & 0,4 \\
\hline $20-4$ matin $\ldots \ldots \ldots$ & 42,5 & 1,6 & 1,6 & 0,3 & 0,4 \\
\hline $20-4$ soir $\ldots \ldots \ldots$ & 42,2 & 1,6 & 1,3 & 0,6 & 0,3 \\
\hline $21-4$ matin $\ldots \ldots \ldots$ & 39,3 & 1,5 & 1,2 & 0,7 & 0,3 \\
\hline $21-4$ soir $\ldots \ldots \ldots$ & 43,2 & 1,7 & 1,3 & 0,7 & 0,3 \\
\hline $22-\varepsilon_{4}$ matin $\ldots \ldots \ldots$ & $41, t_{4}$ & 1,4 & 1,2 & 0,7 & 0,2 \\
\hline $22-4$ soir $\ldots \ldots \ldots \ldots$ & 45,5 & 1,6 & 1,2 & 0,8 & 0,3 \\
\hline $23-4$ matin $\ldots \ldots \ldots$ & 42,4 & 1,3 & 1,2 & 0,7 & 0,3 \\
\hline $23-4$ soir $\ldots \ldots \ldots$ & 42,2 & 1,4 & 1,2 & 0,7 & 0,3 \\
\hline $24-4$ matin $\ldots \ldots \ldots$ & 38,2 & 1,4 & 1,3 & 0,7 & 0,3 \\
\hline $24-4$ soir $\ldots \ldots \ldots \ldots$ & 39,5 & 1,7 & 1,0 & 0,8 & 0,4 \\
\hline $25-4$ matin $\ldots \ldots \ldots$ & 39,1 & 1,6 & 1,2 & 0,7 & 0,3 \\
\hline $25-4$ soir & 40,2 & 1,6 & 1,1 & 0,7 & 0,3 \\
\hline $26-4$ matin $\ldots \ldots \ldots$ & 39,0 & 1,6 & 1,1 & 0,7 & 0,4 \\
\hline $26-\varepsilon_{1}$ soir $\ldots \ldots \ldots$ & 40,4 & 1,8 & 0,9 & 0,7 & 0,4 \\
\hline $27-\varepsilon_{1}$ matin $\ldots \ldots \ldots$ & 41,7 & 1,8 & 1,0 & 0,7 & 0,4 \\
\hline
\end{tabular}


TABLEAU 5

Passage ménagé du régime de stabulation au régime de pâturage. Troupeau $A$.

Variations de l'indice d'iode et des proportious des acides polyinsaturés de la matière grasse du lait.

\begin{tabular}{|c|c|c|c|c|c|}
\hline \multirow{3}{*}{$\begin{array}{c}\text { Dates } \\
\text { des } \\
\text { prélèvements }\end{array}$} & \multirow{3}{*}{ Indice d'iode } & \multicolumn{4}{|c|}{ Acides polyinsaturés (en $\mathrm{g}$ pour $100 \mathrm{~g}$ de matière grasse) } \\
\hline & & \multirow{2}{*}{$\frac{\text { Conjugués }}{\text { Diènes }}$} & \multicolumn{3}{|c|}{ Non conjugués } \\
\hline & & & Diènes & Triènes & Tétraènes \\
\hline $13-4$ matin $\ldots \ldots \ldots$ & 29,8 & 0,6 & 1,0 & 0,4 & 0,3 \\
\hline $13^{-1}$ soir $\ldots \ldots \ldots$ & 33,5 & 0,6 & 1,1 & 0,5 & 0,4 \\
\hline $11_{1}-1_{1}^{\prime}$ matin $\ldots \ldots \ldots$ & 28,6 & 0,4 & 1,0 & 0,4 & $0,4^{\prime}$ \\
\hline $1 t_{1}-4$ soir $\ldots \ldots \ldots$ & 33,5 & 0,6 & 1,1 & 0,5 & 0,3 \\
\hline $15-4$ matin $\ldots \ldots \ldots$ & 30,5 & 0,5 & 1,3 & 0,5 & 0,3 \\
\hline $15-4$ soir...$\ldots \ldots$ & 34,4 & 0,8 & 1,2 & 0,5 & 0,4 \\
\hline $16-1$ matin...$\ldots \ldots$ & 32,4 & 0,7 & 1,1 & - & 0,3 \\
\hline $16-1$ soir $\ldots \ldots \ldots \ldots$ & 37,6 & 1,0 & 1,3 & 0,5 & 0,4 \\
\hline $17-1$ matin...$\ldots \ldots$ & 35,0 & 1,1 & 1,3 & 0,4 & 0,4 \\
\hline $17-4$ soir $\ldots \ldots \ldots$ & 37,5 & 1,3 & 1,3 & 0,6 & 0,4 \\
\hline $18-4$ matin . . . . . & $37, \underline{2}$ & 1,3 & 1,5 & 0,4 & 0,3 \\
\hline $18-1$ soir...$\ldots \ldots$ & 39,9 & 1,6 & 1,2 & 0,5 & 0,3 \\
\hline $19-4$ matin...$\ldots \ldots$ & 37,6 & 1,3 & 1,2 & 0,6 & 0,3 \\
\hline 19-4 soir...$\ldots \ldots$ & 41,7 & 1,6 & 1,3 & 0,6 & 0,3 \\
\hline $20-4$ matin $\ldots \ldots \ldots$ & 39,4 & 1,5 & 1,5 & 0,2 & 0,4 \\
\hline $20-4$ soir $\ldots \ldots \ldots \ldots$ & 42,9 & 1,8 & 1,2 & 0,6 & 0,3 \\
\hline $21-1$ matin ........ & 39,1 & 1,6 & 1,2 & 0,6 & 0,3 \\
\hline $21-4$ soir $\ldots \ldots \ldots$ & 42,1 & 1,9 & 1,0 & 0,8 & 0,3 \\
\hline $20-4$ matin...$\ldots \ldots$ & 41,1 & 1,6 & 1,2 & 0,6 & 0,3 \\
\hline $22-4$ soir $\ldots \ldots \ldots \ldots$ & 42,7 & 1,6 & 1,3 & 0,6 & 0,3 \\
\hline $23-4$ matin ......... & 41,7 & 1,3 & 1,3 & 0,7 & 0,3 \\
\hline $23-4$ soir $\ldots \ldots \ldots$ & 42,4 & 1,5 & 1,5 & 0,7 & 0,4 \\
\hline $24-4$ matin $\ldots \ldots \ldots$ & 37,3 & 1,4 & 1,1 & 0,6 & 0,4 \\
\hline $24-4$ soir $\ldots \ldots \ldots \ldots$ & 39,2 & 1,6 & 1,1 & 0,7 & 0,3 \\
\hline $25-4$ matin ......... & 38,8 & 1,6 & 1,0 & 0,7 & 0,4 \\
\hline $25-4$ soir . . . . . . . & 39,8 & 1,8 & 1,2 & 0,8 & 0,3 \\
\hline $26-4$ matin...$\ldots \ldots$ & 39,4 & 1,5 & 1,2 & 0,7 & 0,3 \\
\hline $26-4$ soir $\ldots \ldots$. & 40,3 & 1,8 & 1,1 & 0,7 & 0,3 \\
\hline $27-4$ matin $\ldots \ldots \ldots$ & 41,5 & 1,8 & 0,9 & 0,7 & 0,3 \\
\hline
\end{tabular}

TABLEAU 6

Proportions moyennes des acides polyinsaturés de la matière grasse des laits de mélange correspondant aux troupeaux $A$ et $B$, du 13 au 27 avril 1959.

(en $g$ pour roo $g$ de matière grasse)

\begin{tabular}{l|c|c|c|c|c}
\hline \hline & Indice d'iode & $\begin{array}{c}\text { Acides diènes } \\
\text { conjugués }\end{array}$ & \multicolumn{2}{|c}{ Acides non conjugués } \\
\cline { 3 - 6 } & & & Diènes & Triènes & Tetraènes \\
\hline $\begin{array}{l}\text { Troupeau A } \\
\text { Sortie aau pâturage } \\
21 \text { avril ............. }\end{array}$ & 37,8 & 1,30 & 1,19 & 0,58 & 0,34 \\
\hline $\begin{array}{l}\text { Troupeau B } \\
\text { Sortie au pâturage : } \\
14 \text { avril .............. }\end{array}$ & 38,4 & 1,29 & 1,21 & 0,56 & 0,34 \\
\hline \hline
\end{tabular}


firmons bien ce point mais seulement après la mise à l'herbe. Avant la mise à l'herbe nous n'observons qu'un maximum très peu prononcé, la courbe se rapproche d'un plateau et ce maximum, si peu marqué soit-il, ne se place pas à 232 mu mais à 226 $228 \mathrm{~m} \mu$. Au moment précis de la mise à l'herbe, ou plus exactement aul cours des quelques jours qui suivent cette mise à l'herbe et simultanément à l'augmentation de la proportion d'acides diènes conjugués, nous observons un déplacement du maximum de $228 \mathrm{~m} \mu$ à $232-233 \mathrm{~m} \mu$. Ceci est mis en évidence sur la figure $6:$ le maximum se place à $228 \mathrm{~m} \mu$ ( 24 avril), puis à $230 \mathrm{~m} \mu$ ( 26 avril), enfin à $232 \mathrm{~m} \mu$ ( 27 avril).

D'après nos calculs la quantité de diènes conjugués correspondant à la courbe du 24 avril $195^{8}$ est de $0,4 \mathrm{~g}$ pour Ioog de matière grasse.

\section{2. - Passage ṁénagé du régime de stabulation au régime de pâturage (I959).}

Les tableaux 4 et 5 donnent l'indice d'iode et les proportions des différents acides poly-insaturés de la matière grasse des beurres correspondant aux trotupeaux $A$ et $B$, fabriqués chaque jour du $I_{3}$ au 27 avril 1959 . Les valeurs moyennes sont portées dans le tableau 6.

On voit que les proportions des différents acides poly-insaturés se rapportant aux troupeaux $A$ et $B$, sont très voisines et parfois même identiques.

a) Acides conjugués (fig. 7)

- Diènes. - Du I3 au 27 avril on note une augmentation de 0,45 à $\mathrm{I}, 8 \mathrm{~g}$ pour Ioo $g$ de matière grasse. Ainsi, comme en 1958 , la proportion d'acides diènes conju-

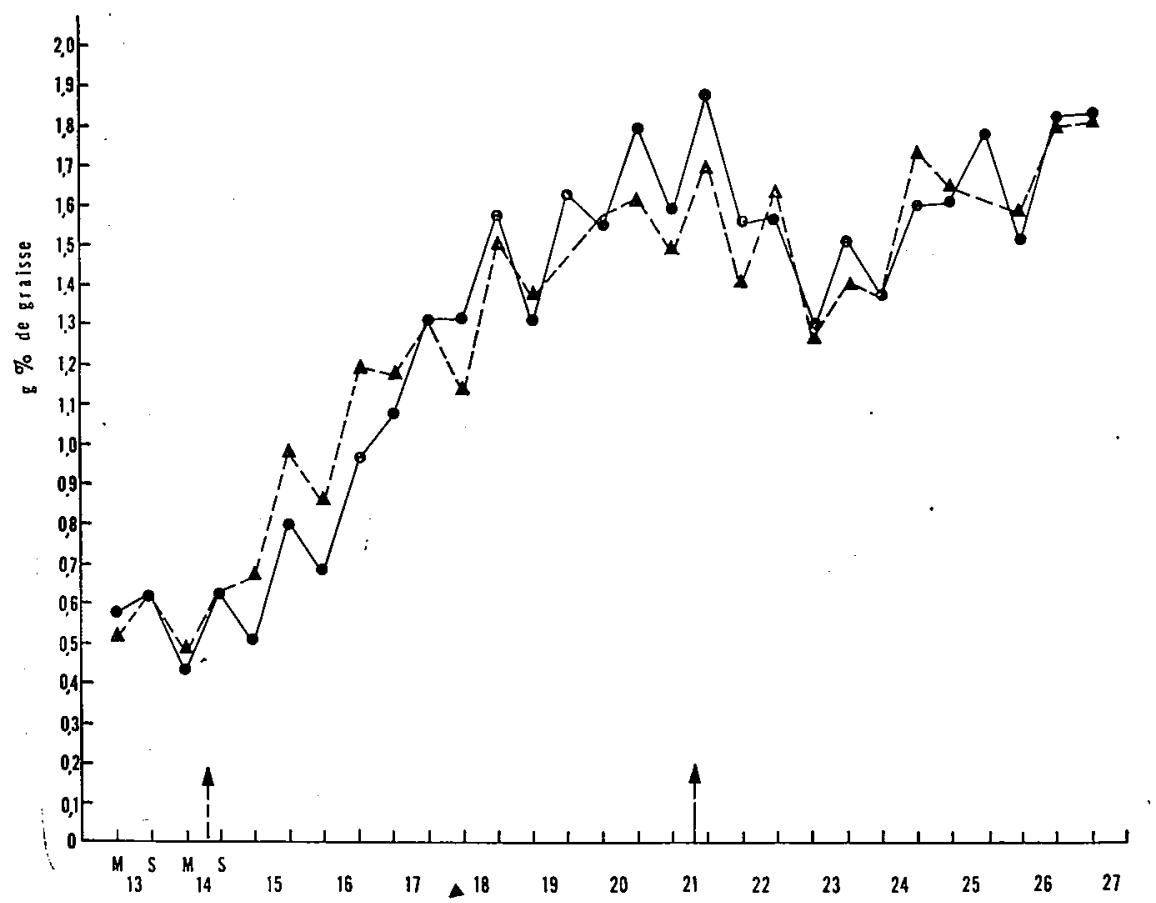

Frg. 7 - Proportions des diènes conjugués dans la matière grasse du beurre, du 13 au 27 avril 1959. 
gués est, après l'établissement du régime de pâturage, approximativement trois fois plus élevée qu'avant le changement de régime alimentaire. L'amplitude des variations de la proportion des acides diènes conjugués est cependant un peu plus grande en I959 qu'en I958 (0,4 à I,9 en I959 contre 0,4 à I,3 en I958, en g pour Ioo $g$ de matière grasse).

Le déplacement du maximum d'absorption vers $232 \mathrm{~m} \mu$ a été de nouveau observé en I959 (fig. 6).

- Triènes et tétraènes - Sur la matière grasse non isomérisée des échantillons de I959 on a observé comme sur la matière grasse des échantillons de I958, un maximum d'absorption à $268 \mathrm{~m} \mu$ et également un maximum à $3 \mathrm{I} 6 \mathrm{~m} \mu$ et non plus comme sur les échantillons de 1958 une simple inflexion de la courbe d'absorption.

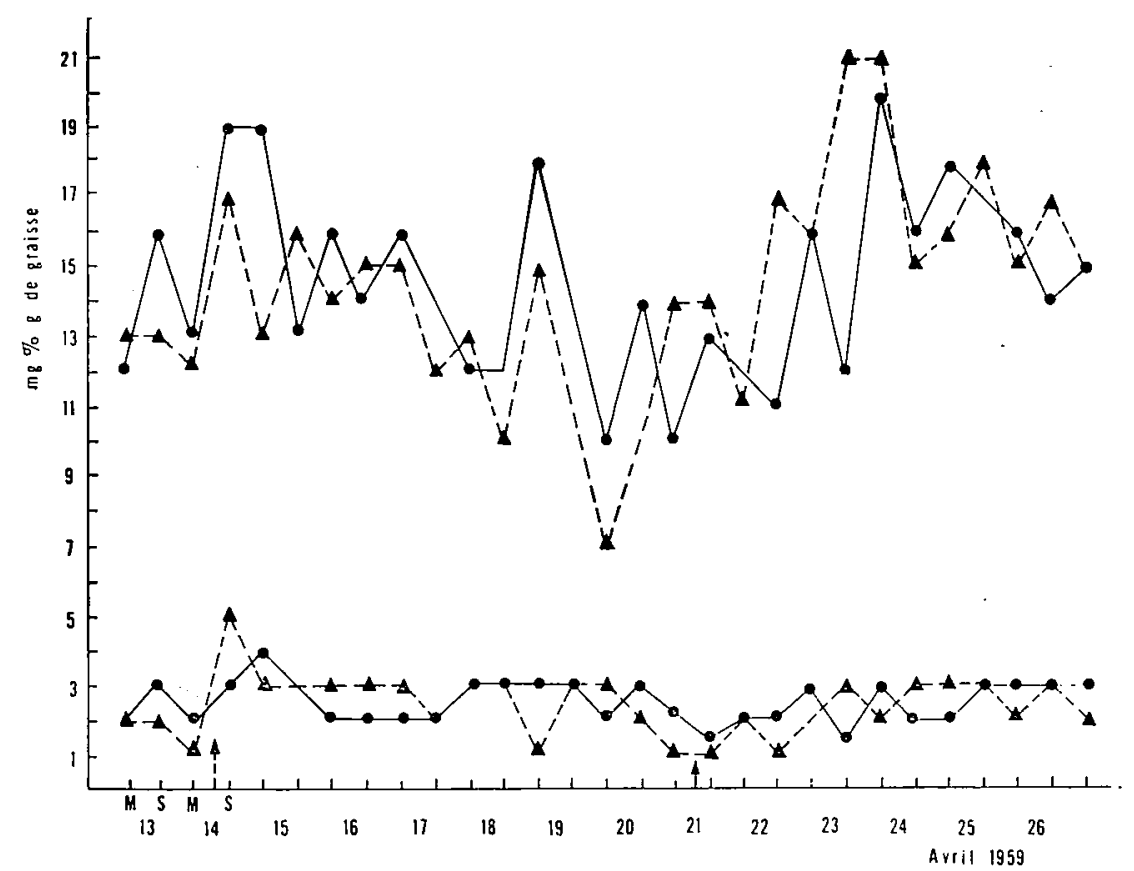

FIG. 8 - Variation du 13 au 27 avril des triènes (graphique supérieur) et des tétraènes (graphique inférieur) conjugués dans la matière grasse du beurre provenant dn troupeau $A$
el du troupeau $B(\mathbf{-})$.

Le calcul effectué suivant BRICE et SwAIN (I945) conduit aux valeurs moyennes suivantes : pour les acides triènes conjugués, o,0I45 et 0,0I43 (en $\mathrm{g}$ pour Ioo $\mathrm{g}$ de matière grasse) respectivement pour les échantillons se rapportant aux troupeaux $A$ et $B$ et pour les acides tétraènes conjugués 0,0025 et 0,0024 (en $g$ pour Ioo $g$ de matière grasse).

Ces valeurs extrêmement faibles ne peuvent avoir qu'une signification limitée. En effet, d'après Brick et Swain (I945), on ne peut espérer obtenir qu'un ordre de grandeur lorsque les quantités d'acides déterminées sont égales ou inférieures à $0, \mathrm{I}$ p. Ioo. Toutefois, les variations des triènes et tétraènes conjugués observées en I959 (fig. 8) sont assez similaires pour les troupeaux A et B. Ceci peut laisser supposer qu'il s'agit de variations réelles existant au niveau des échantillons. 
b) Acides non conjugués (fig. 9)

On observe en 1959 et 1958 une augmentation passagère (vers le milieu de la période expérimentale) de la proportion d'acide linoléique, mais à la fin de l'expérimentation cette proportion revient à sa valeur initiale. Du I3 au 27 avril I959, la proportion moyenne d'acide linoléique est de $\mathrm{I}, 2$ (en $\mathrm{g}$ pour roo g de matière grasse). Cette proportion moyenne était de I,5 du 2 I au 30 avril 1958 .

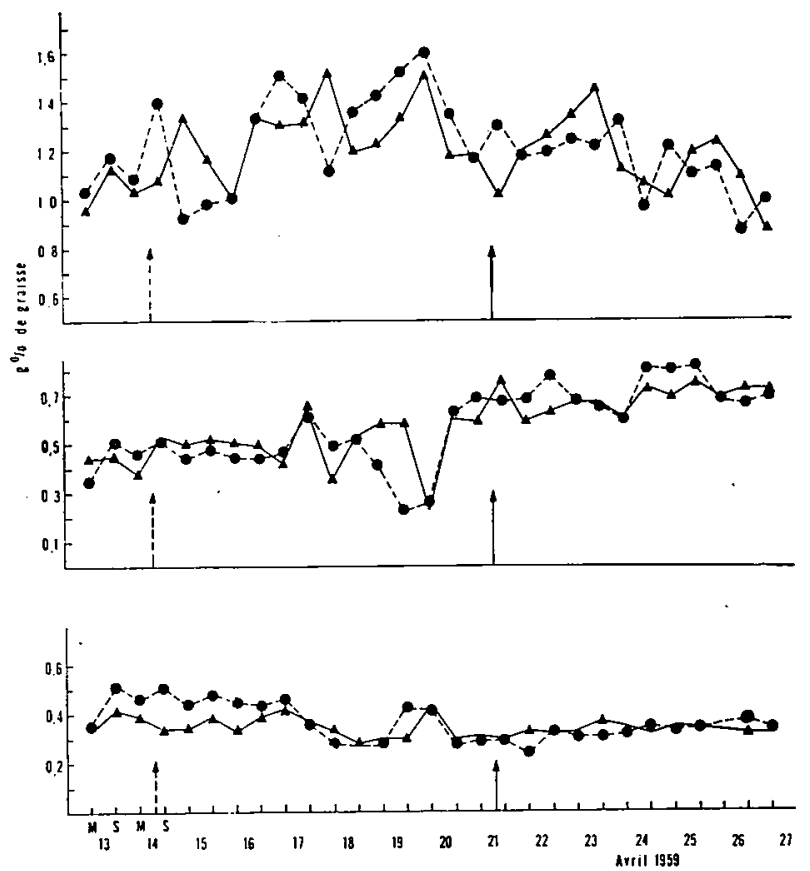

FIG. 9 - Variation des proportions de diènes (graphique supérieur), triènes (graphique central) et tétraènes (graphique intérieur) non conjugués, dans la matière grasse du beurre. du 13 au 27 avril 1959. $\Delta-\Delta$ troupeau $\mathrm{A}$

- - - troupeau B.

La proportion des acides triènes non conjugués (linolénique) atteint après l'établissement du régime de pâturage une valeur approximativement égale au double de la valeur trouvée en période de stabulation (variation de 0,35 à $0,70 \mathrm{~g}$ pour Ioo $\mathrm{g}$ de matière grasse). Les valeurs observées en I958 et I959 sont du même ordre.

Ia proportion moyenne d'acides tétraènes non conjugués observée en 1959 $\left(0,34 \mathrm{~g}\right.$ pour Ioo $\mathrm{g}$ de matière grasse) est très voisine de celle observée en $195^{8}(0,38)$.

\section{DISCUSSION}

\section{A. - COMPOSITION DE LA MATIÈRE GRASSE DU LAIT E'T TAUX BUTYREUX}

Compte-tenu de nos connaissances actuelles concernant la synthèse de 1a matière grasse du lait, les variations des indices, c'est-à-dire en fait les variations de la proportion relative des acides gras, peuvent être le reflet 
de modifications intervenant dans le processus de formation de la matière grasse du lait au niveau des "précurseurs ". En conséquence, il est possible que la quantité de matière grasse secrétée et le taux butyreux du lait soient modifiés pendant la période de changement de régime alimentaire. Tноме (I944) a signalé en Suède le cas d'une baisse très importante du tatıx butyreux (de 3,2 à $I, 4$ p.roo) constatée au début de la période de pâturage sur des laits individuels. L'indice d'iode de la matière grasse des laits dont le taux butyreux était inférieur à 2 p. Ioo, dépassait toujours 50 et s'élevait parfois à plus de 60 .

$\mathrm{Si}$ de tels accidents peuvent être relevés, il semble que très généralement ce soit plutôt une augmentation passagère du taux butyreux que l'on observe, à la mise à l'herbe. En effet, JARRIGE et JOURNE'T (1959) ont déterminé chaque jour, le taux butyreux moyen du troupeau de Jouy-en-Josas pendant la période de changement de régime alimentaire (printemps 1958 ) et ont observé d'abord une baisse de ce tatx le jour même de la mise à l'herbe (baisse due, semble-t-il à une rétention de lait importante) suivie d'une augmentation atteignant Io p. Ioo (par rapport à la veille de la mise à l'herbe) entre le second et le troisième jour après la mise à l'herbe.

\section{B. - VARIATIONS ENTRE IA COMPOSITION DE LA MATIÈRE GRASSE DU MATIN ET LA COMPOSITION DE IA MATIÈRE GRASSE DU SOIR}

Nous avons insisté, dans l'exposé des résultats, sur la différence existant entre l'indice d'iode du soir et celui du matin, le second étant en général inférieur au premier.

Un fait semblable a été rapporté par Thome (I944) et par MAYHEAD et BarnICOAT (I956). Ceux-ci attribuent la différence observée à la manière particulière dont se nourrissent les vaches en pâture, de jour et de nuit. Lorsque de telles variations sont relevées en régime de stabulation, on doit sans doute les imputer à la nature de l'alimentation et à l'horaire de distribution.

\section{C. - Variations des acides poly-insaturés}

\section{I. - Acides diènes conjugués}

La teneur minima que nous relevons avant la mise à l'herbe du troupeau laitier est $0,4 \mathrm{~g}$ pour Ioo $\mathrm{g}$ de matièe grasse. MatTsSon et SwarTLING (1958) signalent la même teneur minima ; STADHOUDERS et MULDER (I955) l'estiment légèrement supérieure $(0,58)$. Ce chiffre correspond-il à une quantité réelle ou doit-on considérer que, le maximum d'absorption étant peu marqué et ne se plaçant pas à $232 \mathrm{~m} \mu$, il n'y a pas de diènes conjugués? En d'autres termes, ce chiffre de 0,4 doit-il être considéré comme égal à zéro ? La proportion réelle de diènes conjugués dans le beurre serait alors égale aux valeurs consignées dans les tableaux 2,4 et 5 , diminuées de 0,4 .

Nous avons montré l'existence d'un déplacement du maximum d'absorption de 228 à $232 \mathrm{~m} \mu$, avant isomérisation. Ce déplacement du maximum apparait également dans une étude poursuivie par Holm et WODE (1949) sur l'oxydation comparée d'un beurre d'été et d'un beurre d'hiver. L'étude minutieuse des graphiques présentés par ces auteurs (fig. I et 2 de leur publication), permet d'affirmer qu'un déplacement du maximum de quelques unités vers les longueurs d'onde du spectre 
visible a été observé simultanément à l'augmentation progressive de l'absorption au cours de l'oxydation du beurre d'hiver. Par contre au cours de l'oxydation du beurre d'été un tel phénomène n'a pas été relevé, le maximum d'absorption étant toujours net et se plaçant toujours à la même longueur d'onde de $232 \mathrm{~m} \mu$. Les peroxydes formés (conjugués) ont vraisemblablement (PAQUoT, I959) une structure cis-trans et trans-trans. Le maximum d'absorption de l'isomère trans comparé avec celui de l'isomère cis étant en général déplacé de 2,5 à $5 \mathrm{~m} \mu$ vers le spectre visible (DEUEL, I950) on pourrait ainsi expliquer le déplacement du maximum d'absorption au cours de l'oxydation du beurre d'hiver.

Quelle est l'origine du déplacement du maximum d'absorption que nous avons nous-mêmes observé? L'introduction d'herbe dans l'alimentation des vaches laitières apporte une quantité considérable d'acide linolénique au niveau du rumen. En effet, cet acide représente environ $60 \mathrm{p}$. Ioo des acides gras de l'herbe (SHorland, WeEnink et Johns, I955) (Hartuan, Shorland et Moir, 1956). Il possède une structure cis, cis, cis puisqu'il a été montré que les acides trans n'existaient qu'à l'état de traces dans les lipides de l'herbe (Hartman, Shorland et McDonald, r954). Or cet acide n'apparait que dans une faible proportion (0,5 p. roo) dans la matière grasse du beurre. Il est en effet hydrogéné au niveau du rumen. SHORLAND, WEENINK et JoHNS (I955) ont montré que l'hydrogénation comporte les étapes suivantes : formation de diènes conjugués, d'acide monoène et d'acide stéarique ; comme au cours de toute hydrogénation on observe simultanément la formation d'acides trans (Hartman, Shorland et McDonald, I954). SMitrm et Jack (I954) ont d'ailleurs montré que les acides diènes conjugués de la matière grasse du beurre présentaient principalement la configuration cis-trans.

Cependant, seule une étude spectrophotométrique dans la région infra-rouge du spectre permettra de préciser si l'on se trouve en présence d'une augmentation des acides diènes conjugués à structure cis-trans ou trans-trans dans la matière grasse du beurre, lors de la mise à l'herbe des vaches laitières.

\section{2. - Acides diènes non conjugués ou acide linoléique}

La variation de l'acide linoléique ne s'aligne ni sur celle de l'indice d'iode, ni sur celle des diènes conjugués. SHORLAND, WEENINK et Johns (I955) ont d'ailleurs montré que l'acide linoléique ne constitue pas un intermédiaire, dans le phénomène d'hydrogénation au niveau du rumen comme l'avait cependant pensé REISER (I95r). MáTTSON, THOME et SwarTLING (I95 I $b$ ) ont d'autre part montré la grande stabilité de la teneur en acide linoléique du beurre au cours de l'année, ceci dans les conditions normales d'alimentation des vaches laitières. En effet si de l'huile de soja, riche en acide linoléique est introduite dans l'alimentation des vaches laitières, la teneur du beurre en acide linoléique est nettement augmentée (MaTtsson, Thome et SwarTLING, I95I $a$ ).

\section{3. - Acides triènes non conjugués}

Les triènes non conjugués (acide linolénique) augmentent après la mise à 1'herbe. L'acide linolénique étant le principal constituant des acides gras de l'hertc, l'hydrogénation au niveau du rumen n'est sans doute pas totale : wne faible partie de l'acide 
linolénique se retrouve dans la matière grasse du lait. Scorr et al. (I959) ont confirmé que cet acide garde dans la matière grasse du beurre, sa structure propre : cis, cis, cis.

\section{4. - Existence d'acides pentaènes non conjugués}

Au cours de l'expérience menée au printemps 1959 nous avons régulièrement mesuré l'absorption à $348 \mathrm{~m} \mu$ et nous avons régulièrement observé la présence d'un maximum d'absorption à cette longueur d'onde. SHORLAND et JoHANNESSON (I95I), SMrth et JACK (1954) et MATTSSON et SWARTIING (I958) ont attribué ce maximum à la présence, dans la matière grasse du beurre, d'acides pentaènes.

\section{D. - VARIATION DE L'ACIDE OIÁIQUUE}

Théoriquement on peut, connaissant 1'indice d'iode de la matière grasse et les pourcentages des différents acides polyinsaturés; déduire le pourcentage d'acide oléique. C'est de cette façon, croyons-nous, que Stadhouders et Mulder (I955) d'une part, McDowELI, (I953) d'autre part ont calculé le pourcentage d'acide oléique. Nous ne ferons pas, pour notre part, ce calcul. S'agirait-il en effet uniquement d'acide oléique (cis 9, octadecenoïque)? Puisque l'hydrogénation de l'acide linolénique conduit à un monoène, cet acide monoène ne serait-il pas un isomère trans de l'acide oléique? Récemment, par analyse spectrophotométrique en infra-rouge, CORNWELI, et al. (I953) ont précisé que I4 à $20 \mathrm{p}$. Ioo de l'acide " oléique " du beurre était sous forme trans (mélange probable d'acide vaccénique et d'autres isomères trans de l'acide oléique. )

\section{E. - TENEUR EN ACIDES GRAS POLY-INSATURÉS ET CONSERVATION ULTÉRIEURE DU BEURRE}

Comme l'a récemment rappelé BúJAmbes (I959) l'altération du beurre par autoxydation représente à l'heure actuelle un des problèmes majeurs de la beurrerie. Toute information nouvelle concernant la composition, la nature et les propriétés particulières des acides gras insaturés présente donc un intérêt pratique certain.

Mat'rsson, Thome et Swartling (I94I $a$ ) ont montré que plus le taux des acides en $\mathrm{C}_{18}$ (acide diène conjugué ou acide linoléique) est élevé dans le beurre, plus brève est la période d'induction et plus rapide la vitesse d'oxydation.

Cependant les peroxyde: étant sans odeur (HoL, r, 1949) il est certain que les défauts de goût qui peuvent être provoqués par l'oxydation de la matière grasse au cours de la conservation du beurre à la température ambiante ou à basse température n'apparaissent que lorsque des réactions ultérieures se produisent. Ainsi, Holm, WoDE et Thome (I952) ont montré que le goût " huileux » du beurre, qui sous sa forme accentuée s'identifie au goût " huile de baleine " ou de " poisson ", est provoqué par la présence de composés carbonylés $(\alpha$ et $\beta$ ) insaturés provenant de la décomposition des peroxydes. Ces auteurs distinguent le goût " huileux " du beurre (oily flavour) du défaut de goût connu sous le nom de "goût métallique " et spécifique des laits d'hiver.

On a signalé d’autre part (PAQuoT, I959) que l'acide octadécadiène- IO-I2 oïque, 
isomère de l'acide linoléique, s'oxyde plus lentement que l'acide linoléique 9-I2. Il serait donc du plus haut intérêt, pour l'étude de l'oxydation du beurre, de connaître la place des doubles liaisons des acides conjugués diènes du beurre.

Rę̧u en avril 1960.

\section{SUMMARY}

INFLUENCE OF TURNING DAIRY-COWS OUT TO GRASS ON THE INDICES AND THE QUANTITY OF TIIE VARIOUS POLYUNSATURATED FATTY ACIDS IN THE BUTTER FAT.

The fat of butter made from the morning and evening milk of different groups of cows in the herd at the C. N. R. Z. was studied over a period of a fortnight during which the cows were turn out to grass, in the spring of 1957,1958 and 1959.

Variations in the iodine value and saponification values and the soluble and insoluble volatile fatty acids of the butter fat were recorded in 1957 , whereas in $195^{8}$ and I959 only the variations in iodine value were studied with additional determination of the various poly-unsaturated (conjugated and non-conjugated) fatty acids in the butter fat.

The increase in iodine value which occurs when fresh grass is introduced into the cow's diet may be abrupt (I2 units in 48 hours) or less rapid depending on whether the change from the winter ration to pasture is sudden or gradual.

There is a correlated increase in the amount of conjugated diene acids (expressed as percentage by weight of butter fat), with the increase in iodine value (from 0,5 to 1,3 in 1958 and 0,5 to 1,8 in 1959 for a corresponding increase of 14 units in the iodine value). A simultaneous increase in linolenic acid $(0,3$ to 0,8$)$ is observed.

There is a transitory increase in the amount of linoleic acid which returns to its initial value several days after the cows are put out to grass ( $\mathrm{I}, 5$ in 19.58 and only $\mathrm{I}, 0$ in $\mathrm{I} 959$ ). There is no variation in the amount of arachidonic acid $(0,3$ to 0,4$)$.

\section{RÉFÉRENCES BIBLIOGRAPHIQUES}

Bejambes M., i 959. La conservation du beurre. L'oxydation. Ann. Nul. Alim., 13, A $28 \mathrm{r}-\mathrm{A} 297$.

BEJAMBES H., SAvore S., i954. Étude comparée des variations saisonnières de l'indice d'iode des beurres en Normandie et en Alsace. Chim. Industr., r1, 50I-506.

BERGmax I., Joost K., I953. The iodine value of milk fat during different parts of the year, and its influence on the quality of the butter. XII Intern. Dairy Cong. La Haye, 2, 89-92.

BRICE B. et SwAIN II., I945. Ultraviolet absorption method for the determination of polyunsaturated constituents in fatty materials. J. Opt. Soc. Amer., 35, 532-544.

Cornwell D., Backderf R., Wilson C., et Brown J., I953. The trans-octadecenoic acid content of butter fat. Arch. Biochen. Biophys., 46, 364-375.

Cox G., Mc Dowall F., I948. The properties of New Zeeland butters and butter fats. I. Iodine, Reichert and saponification values and softening points of monthly samples of butter fats from nine commercial factories over four years. J. Dairy Res., 15, 377-386.

Deuel H., I95r. The lipids. Inlerscience pnbishers inc. New York.

MC Dowell A., I953. The properties of New-Zealand butters and butter fats. III. Seasonal variations in the nature of the unsaturated acids of butter fat as estimated by spectrophotometric methods. J. Dairy Res., 20, IOI-IO7.

HANSEN R., SHORLAND F., I952. Seasonal variations in fatty acid composition of New Zealand butterfat. Biochem. J., 52, 207-216.

Hartman L., Shorland F., Mc Donald I., I954. Occurrence of trans-acids in animal fats. Nalure, 174, I 85 .

HARTMAN L., Shorland F., MoIR R, I956. Occurrence of trans-unsaturated fatty acids in horse faeces. Nature, 178, 1057.

HoLM U., I949. The relationship of peroxyde values to tallowy flavors in various fats. XII Intern. Dairy Cong. Stockholm, II, 336-340.

Holm U., WODE G., I 949 . Spectrophotometric investigation of oxidised butter fat. XIIe Intern. Dairy Cong. Stockholm, II. 325-335.

HoLm U., WODE G., THOME K., I952. Oily flavour in butter and $\alpha$, $\beta$-unsaturated carbonyl compounds. Medd. Mejeriförs. Malmo., 37, is pp.

JAMótTe P., PIRAux E., I957. Indices de réfraction et caractéristiques de consistance des beurres belges, Bull. Inst. Agron. Gembloux, 25,:297-328. 
JARRIGE R. et JOURNET M., I959. Influence des facteurs alimentaires et climatiques sur la teneur en matières grasses du lait,. Ann. Nut. Alim., 13, A 233 - A 277 .

Jensien H., Pedersen A., OvergaARd S., I958. Examinations relating to the use of Jersey milk for production of butter. Beretn. Forsgs mej., 113, $5 \mathrm{I}$ pp.

KuZDZAL-SAvole S., I $959 a$. Influence de la race des vaches laitières sur la composition de la matière grasse du lait et la qualité du beurre. Ann. technol. agr., 1, 35-53.

KuzDzAL-SAvoie S., I $959 b$. Influence de la race des vaches laitières sur la composition de la matière grasse du lait.. Ann. Technol. Agr., 2, r93-222.

LembKe A., KaufmanN W., I954. UUber den Gehalt des Milchfettes an Konguensauren. Milchwissenschafl, 9, II 3 -II4.

MATTSSON S., SWARTLING P., 1958. Note on the spectrophotometric determination of polyunsaturated fatty acids in butter. Report 55. Dairy Department of the Alnarp Institule. Suède.

MATTSSON S., Thome K., Swartuing P., I95 I $a$. Polyunsaturated fatty acids in butter and their influence on the oxidation of butter. 2. Oxidation of butter fat of different composition. Medd. Mejeriförs. Malmo, $35,20 \mathrm{pp}$.

PAQUOT C., 1959. L'altëration des corps gras alimentaires. Acidification spontanée, ‘utoxydation, rancissement, réversion. Ann. Null. Alim., 13, A 298 - A 312.

Reiser R., I95I. Fed Proc., 10, 236. cité par ShORLAND F. et al., r955.

Schwarz G., Ludwig J. et GIEssler E., 1957. Eingenschaften und Zusammensetzung von Kuhmilch in Baden-Württemberg. Milchw. Forsch. Kiel, 3, I4 pp.

ScotT W. E., Herb S. F., Magidman P., Riemenschneider R. W., r959. Insaturated fatty acids of butter fat. J. Agric. Food Chenl., Y, 125-I29.

Silorland F. ,Johannesson D., I95I. $\mathrm{C}_{20-22}$ unsaturated acids of butterfat. Nature, 168, 75.

Shorland F., WeEnink K., Johns A., I955. Effect of the rumen on dietary fat. Nature, 175, i 29.

SMITH L., JACK E., I 954 a. The unsaturated fatty acids of milk fat. II. Conjugated and nonconjugated constituents. J. Dairy Sci., 37, 390-398.

SMITH L., JACK E., I954 b. The unsaturated fatty acids of milk fat. III. Geometrical isomerism. J. Dairy Sci., 37, 399-406.

Stadhouders J., Mulder H., I955. The composition of dutch butterfat. I. Seasonal variations in the unsaturated fatty acid composition of butter fat. Neth. Milk. Dairy J., 9, I82-I93.

Stadhouders J., Mulder H., $195^{6}$. The composition of dutch butterfat. II. Seasonal variations in the saturated fatty acid composition of butterfat. Neth. Milk. Dairy J., 10, 53-59.

STEEN K., I956. Some studies on Danish butterfat. XIV Intern. Dairy Cong. Rome II. (I) $438-447$.

Swartling P., MatTsson S., 1956. On the composition and properties of Jersey milk. Medd. Mejeriförs. Malmo, $47,40 \mathrm{pp}$.

THOME K., I944. A case of great depression of the fat content of the milk at the beginning of the grazing period. Medd. Mejeriförs. Malmo, I4, r39-149.

WOOD F., I956. Seasonal and regional variations in some chemical and physical properties of Alberta butterfat. Canad. J. Agric. Sci., 36, 422-429.

WOOD F., HAAB W., 1957. Seasonal and regional variations in the unsaturated acids of Alberta butterfat. Canad. J. Anim. Sci., 37, I-7.

Zehren V., JaCKson H., I956. A survey of United States butterfat constants. I. Reichert Meissl, Polenske and refractive index values. J. Ass. Ofl. Agric. Chem. Wash., 39, r94-212. 